\title{
Prospection Hydrogéologique et Géophysique du Système Aquifère du Plateau de Mbé, District de Ngabé, Congo
}

Essouli Olivier Florent, (Docteur, Maître-Assistant)

Université Marien NGOUABI, Faculté des Sciences et Techniques, Laboratoire de Géosciences, Brazzaville, Congo

Matini Laurent (Docteur, Maître de Conférences)

Université Marien NGOUABI, Ecole Normale Supérieure,

Laboratoire de Chimie, Brazzaville, Congo

Miyouna Timothée (Docteur, Maître-Assistant)

Université Marien NGOUABI, Faculté des Sciences et Techniques,

Laboratoire de Géosciences, Brazzaville, Congo

Fouroux Momo Amarèze, Doctorant

Université Marien NGOUABI, Faculté des Sciences et Techniques,

Laboratoire de Géosciences, Brazzaville, Congo

\section{Boudzoumou Florent, Professeur Titulaire}

Université Marien NGOUABI, Faculté des Sciences et Techniques, Laboratoire de Géosciences, Brazzaville, Congo

Institut National de Recherches en Sciences Naturelles et Exactes,

Brazzaville, Congo

Faye Serigne, Professeur Titulaire

Université Cheikh Anta Diop, Faculté des Sciences et Techniques,

Département de Géologie, Dakar-Fan, Sénégal

Doi:10.19044/esj.2019.v15n30p357 URL:http://dx.doi.org/10.19044/esj.2019.v15n30p357

\section{Resume}

La prospection hydrogéologique et géophysique entreprise sur les sites des villages d'Imvouba et d'Ingah dans le plateau de Mbé a permis de déterminer la qualité des eaux souterraines et de surface ainsi que les niveaux aquifères favorables à l'implantation des forages productifs. La prospection géophysique utilisant les méthodes des résistivités électriques avec le dispositif Schlumberger est menée dans le but de déterminer les emplacements favorables à l'implantation des forages positifs avec des débits d'exploitation importants pour un approvisionnement en eau potable des populations. Cette prospection géophysique a révélé que la foration devrait se faire à des profondeurs prévisionnelles de 200 à $250 \mathrm{~m}$ voir plus afin d'atteindre les débits 
prévus par le projet. La prospection hydrogéologique des eaux souterraines et de surface a montré la dominance du faciès chimique bicarbonaté et calcique et que la bonne qualité physico-chimique des eaux de la région est remise en cause par la présence de certains éléments comme le fer total, le manganèse, les phosphates et l'aluminium à des teneurs élevées dépassant parfois les concentrations maximales admissibles pour l'eau de boisson (OMS, 2017). Les formations géologiques présentes dans le plateau de Mbé renferment un système aquifère multicouche bien alimentés par l'infiltration directe des eaux de pluie (1600 à $2500 \mathrm{~mm} / \mathrm{an})$.

Mots-clés: Hydrogéologie, Prospection géophysique, Sondage électrique, Aquifère, Forage, Mbé 


\section{Hydrogeological and Geophysical Prospecting of the Aquifer System of the Mbe Plateau at Ngabe District, Congo}

Essouli Olivier Florent, (Docteur, Maître-Assistant)

Université Marien NGOUABI, Faculté des Sciences et Techniques, Laboratoire de Géosciences, Brazzaville, Congo

Matini Laurent (Docteur, Maître de Conférences)

Université Marien NGOUABI, Ecole Normale Supérieure,

Laboratoire de Chimie, Brazzaville, Congo

Miyouna Timothée (Docteur, Maître-Assistant)

Université Marien NGOUABI, Faculté des Sciences et Techniques,

Laboratoire de Géosciences, Brazzaville, Congo

Fouroux Momo Amarèze, Doctorant

Université Marien NGOUABI, Faculté des Sciences et Techniques,

Laboratoire de Géosciences, Brazzaville, Congo

\section{Boudzoumou Florent, Professeur Titulaire}

Université Marien NGOUABI, Faculté des Sciences et Techniques, Laboratoire de Géosciences, Brazzaville, Congo

Institut National de Recherches en Sciences Naturelles et Exactes,

Brazzaville, Congo

Faye Serigne, Professeur Titulaire

Université Cheikh Anta Diop, Faculté des Sciences et Techniques,

Département de Géologie, Dakar-Fan, Sénégal

\section{Abstract}

The hydrogeological and geophysical prospection carried out on the sites of the Imvouba and Ingah villages in the Mbé plateau has made it possible to determine the quality of the groundwater and surface water as well as the aquifer levels, which is favorable to the implantation of the productive boreholes. Geophysical prospecting, based on electrical resistivity methods using the Schlumberger device, was conducted in order to determine the favorable locations for the implantation of positive boreholes with high exploitation rates for a drinking water supply of the populations. This geophysical survey revealed that the drilling should be done at predicted depths of 200 to $250 \mathrm{~m}$ or more in order to reach the projected flows of the project. Hydrogeological prospecting of groundwater and surface water has 
shown the dominance of the bicarbonated and calcic chemical facies. The good physicochemical quality of the waters of the region shows the presence of some elements such as total iron, manganese, phosphates and aluminum at high levels which sometimes exceeds the maximum permissible concentrations for drinking water (WHO, 2017). The geological formations present in the Mbé plateau contain a multilayer aquifer system well fed by the direct infiltration of rainwater (1600 to $2500 \mathrm{~mm} /$ year).

Keywords: Hydrogeology, Geophysical prospecting, Electrical resistivity, Aquifer, Drilling, Mbé

\section{Introduction}

Le secteur de l'eau en République du Congo représente de nos jours une priorité nationale et occupe une place de choix dans le cadre stratégique de l'amélioration des conditions de vie des populations du milieu rural. Le pays connait cependant des insuffisances en matière de gouvernance de l'eau aussi bien au niveau central que local. Actuellement, de nombreuses incertitudes subsistent dans les chiffres de taux de couverture et de desserte en eau potable. Différentes études expliquent et montrent l'importance de la prospection hydrogéologique et géophysique afin d'améliorer l'accessibilité des populations à une eau de qualité et en quantité suffisante. Parmi les méthodes utilisées pour l'exploration des eaux souterraines, on note la technique de résistivité électrique largement utilisée (Mohamaden \& Ehab, 2017 ; Abdel Galil et al., 2015 ; Anechana et al., 2015 ; Selim, 2009).

Une campagne de prospection hydrogéologique et géophysique a été réalisée dans les villages Imvouba et Ingah dans le district de Ngabé (Département du Pool) en vue de l'implantation des forages d'eau avec une précision maximale. Les populations de cette partie du pays s'approvisionnent par l'intermédiaire des eaux de surface et des eaux de pluies n'ayant pas fait l'objet d'un contrôle sanitaire, ce qui les expose à de multiples problèmes de santé publique, surtout à une période où l'impact de la qualité de l'eau de boisson sur la santé des populations n'est plus à démontrer (Steven et al., 2008).

En outre, la méconnaissance des ressources en eau du plateau de Mbé, la rareté des points d'eau (puits traditionnels et forages) en surface, la mauvaise qualité des eaux qu'on y trouve et leur tarissement en saison sèche, rendent indispensable l'exploitation des eaux souterraines. Il s'agit donc à travers cette étude, de trouver des ressources en eau souterraine en quantité suffisante et de qualité satisfaisante, de localiser et d'identifier les niveaux aquifères productifs favorables à l'implantation des forages d'eau avec des débits d'exploitation soutenus pendant le cycle hydrologique, ceci afin d'améliorer les conditions d'accès à l'eau des populations du plateau de Mbé. 


\section{Materiel et Methodes}

\subsection{Cadre général de la région étudiée}

Le plateau de Mbé est situé sur la route nationale n² dans la partie nord du département du Pool. Il couvre une superficie de $13000 \mathrm{~km}^{2}$, délimité au nord par la rivière Léfini, à l'est par le fleuve Congo, au sud par la bordure méridionale du plateau de Mbé, à l'ouest par la limite occidentale du parc naturel du PPG (Projet Protection des Gorilles) dans la zone sud de la réserve de chasse de la Léfini (Figure 1).

Ce plateau est un grand ensemble hydrogéologique des plateaux Batéké compris entre $3^{\circ}$ et $4^{\circ}$ de latitude Sud et entre $15^{\circ}$ et $16^{\circ} 15^{\prime}$ de longitude Est (Bauer et al., 2015). 

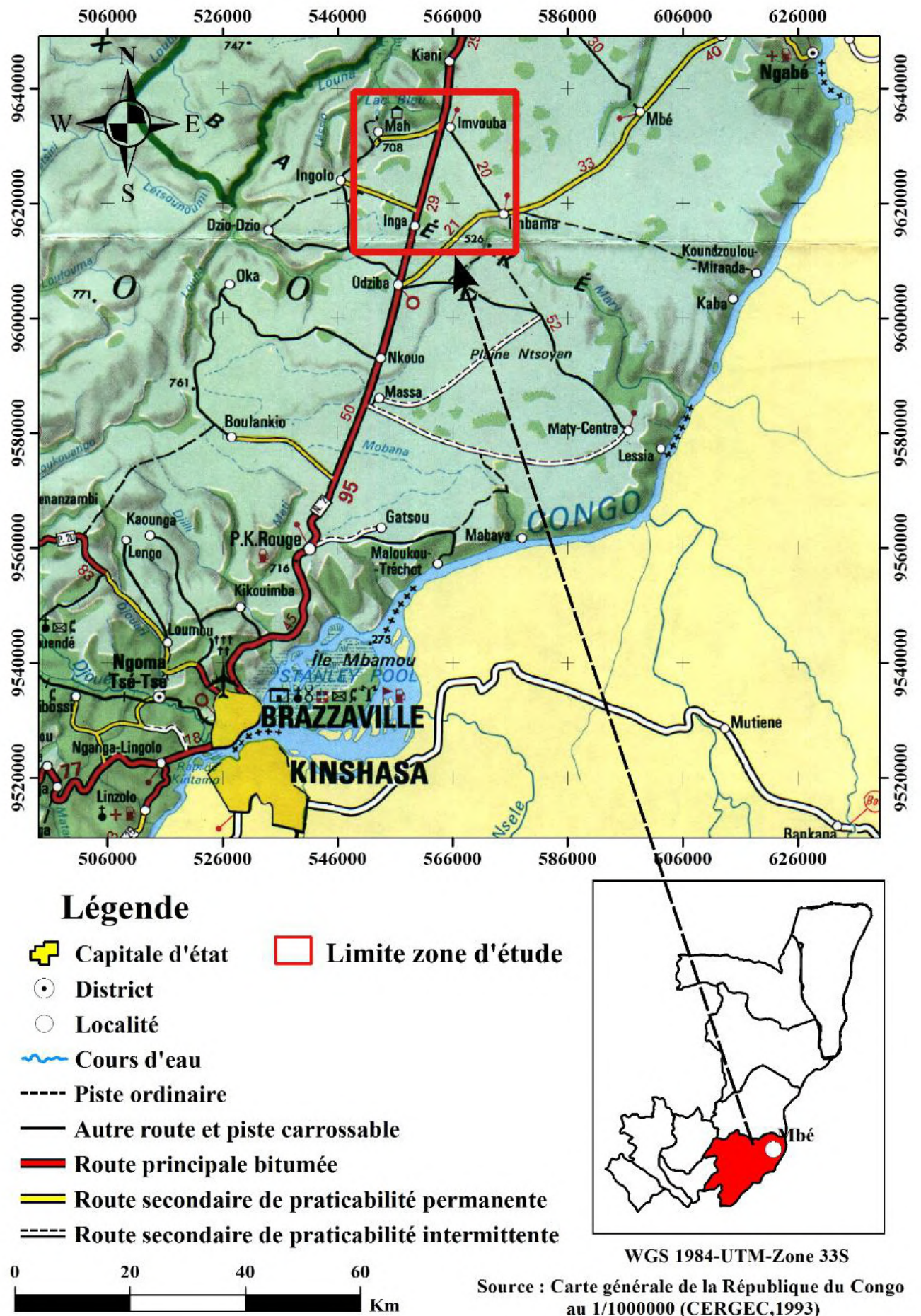

Source : Carte générale de la République du Congo au 1/1000000 (CERGEC,1993)

Figure 1. Situation géographique de la zone d'étude. 


\subsubsection{Climatologie}

Le climat de la région des Plateaux est de type subéquatorial, avec deux saisons principales : une saison des pluies d'octobre à fin avril (interrompue par une période un peu plus sèche entre janvier et février) et une grande saison sèche, de mai à mi-septembre. Dans le secteur d'étude, les précipitations annuelles vont de l'ordre de $1300 \mathrm{~mm}$ au SE de la feuille à $1700 \mathrm{~mm}$ au NW et au centre du plateau. Les températures sont plus élevées en moyenne le long du fleuve Congo et de la Léfini $\left(25,6^{\circ} \mathrm{C}\right.$ annuelle) que sur le plateau de Mbé $\left(23,2{ }^{\circ} \mathrm{C}\right.$ annuelle) (Bauer et al., 2015).

Dans le but de mieux cerner les caractéristiques climatiques de la région d'étude, on s'est référé aux données climatiques de l'ANAC collectées à partir des stations synoptiques de Brazzaville et de Djambala encadrant la zone étudiée sur une période de 46 ans (Figure 2).

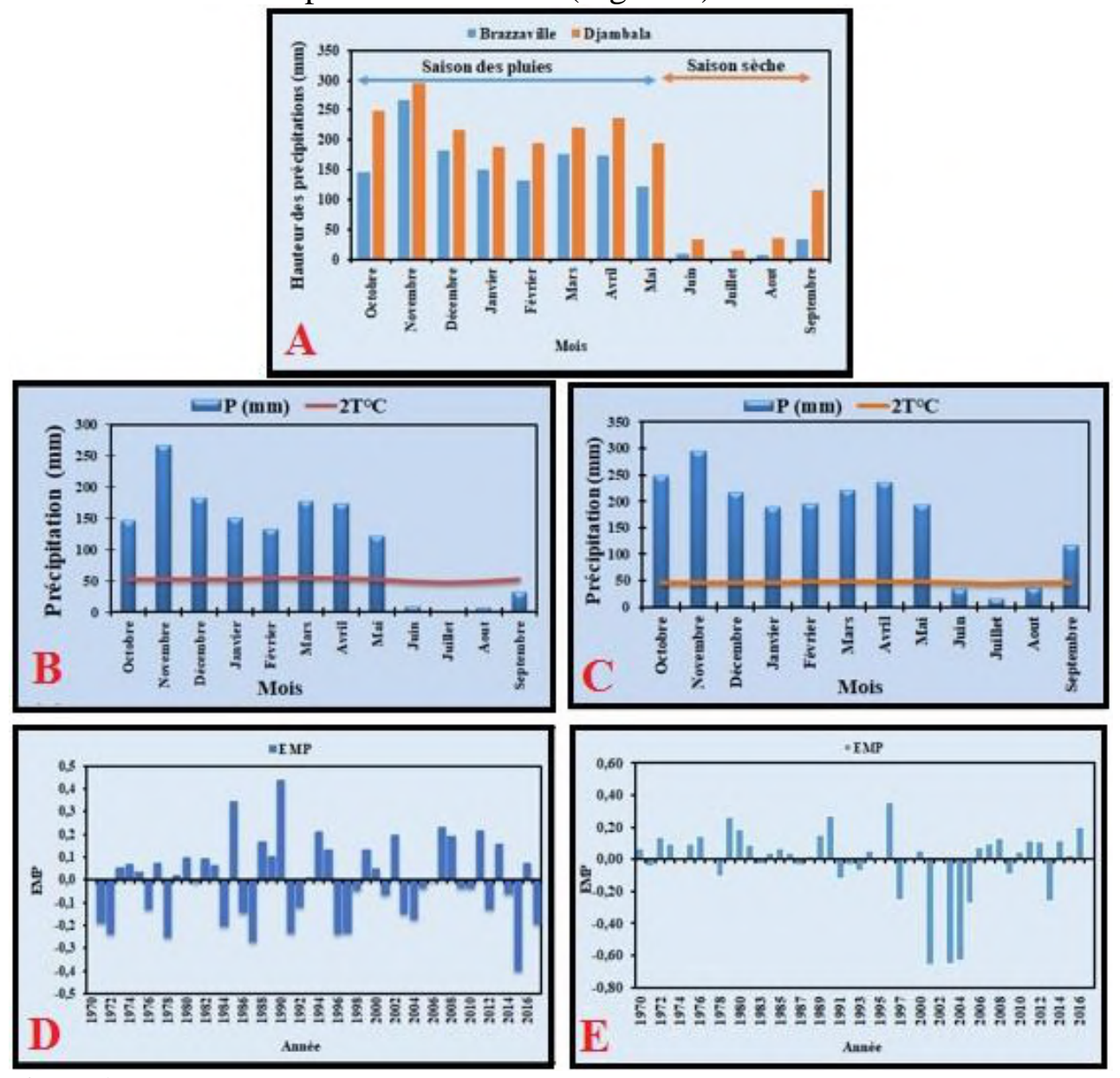

Figure 2. (A) Variation des précipitations moyennes mensuelles aux stations de Brazzaville et de Djambala. (B et C) Diagrammes ombrothermiques des stations de Brazzaville et Djambala. (D et E) Pluviométrie interannuelle aux stations de Brazzaville et Djambala (ANAC, 1970-2016). 
A l'échelle mensuelle, la Figure $2 \mathrm{~A}$ présente la répartition des précipitations moyennes mensuelle. Les deux principales stations synoptiques encadrant le plateau de Mbé (Brazzaville et Djambala) montrent que les précipitations sont abondantes sur une période de huit (8) mois : d'octobre à mai, caractérisant une saison pluvieuse. Cependant, sur les quatre autres mois (juin à septembre), les pluies sont rares et correspondent à une saison sèche marquée par une diminution de la pluviométrie.

Les diagrammes ombrothermiques au niveau des stations synoptiques de Brazzaville et Djambala, montrent que les précipitations sont abondantes sur une période de huit (8) mois d'octobre à mai, caractérisant une saison pluvieuse et la saison sèche qui s'étend du mois de juin à septembre (Figure 2B et C). L'évolution de la pluviométrie interannuelle au niveau des stations synoptiques de Brazzaville et de Djambala montre que les précipitations annuelles mettent en évidence les périodes de pluviosité déficitaires et excédentaires qui diffèrent en fonction des années sans qu'il y ait apparition d'un cycle hydrologique (Figure 2D et E).

\subsubsection{Contexte géologique}

La région des plateaux Batéké a déjà fait l'objet de nombreuses études géologiques (Moukolo et al., 1992 ; Desthieux, 1993). Les plus anciennes d'entre elles datent de 1933 avec Babet qui a reconnu les principales caractéristiques de la série sédimentaire. Cosson en 1955, a cartographié la région au 1/500 000 et, a établi une échelle stratigraphique locale qui comprend de bas en haut (Figure 3) : la série du Stanley-Pool et la série des plateaux Batéké.

La série du Stanley-Pool affleure aux alentours de Brazzaville (Dadet, 1969 ; Giresse, 1990). Elle repose en discordance de ravinement sur le grès de l'Inkisi d'âge permien et comprend $\mathrm{du}$ bas vers le haut trois unités lithostratigraphiques suivantes (Cosson, 1955 ; Giresse, 1990 ; Moukolo et al., 1992) : 


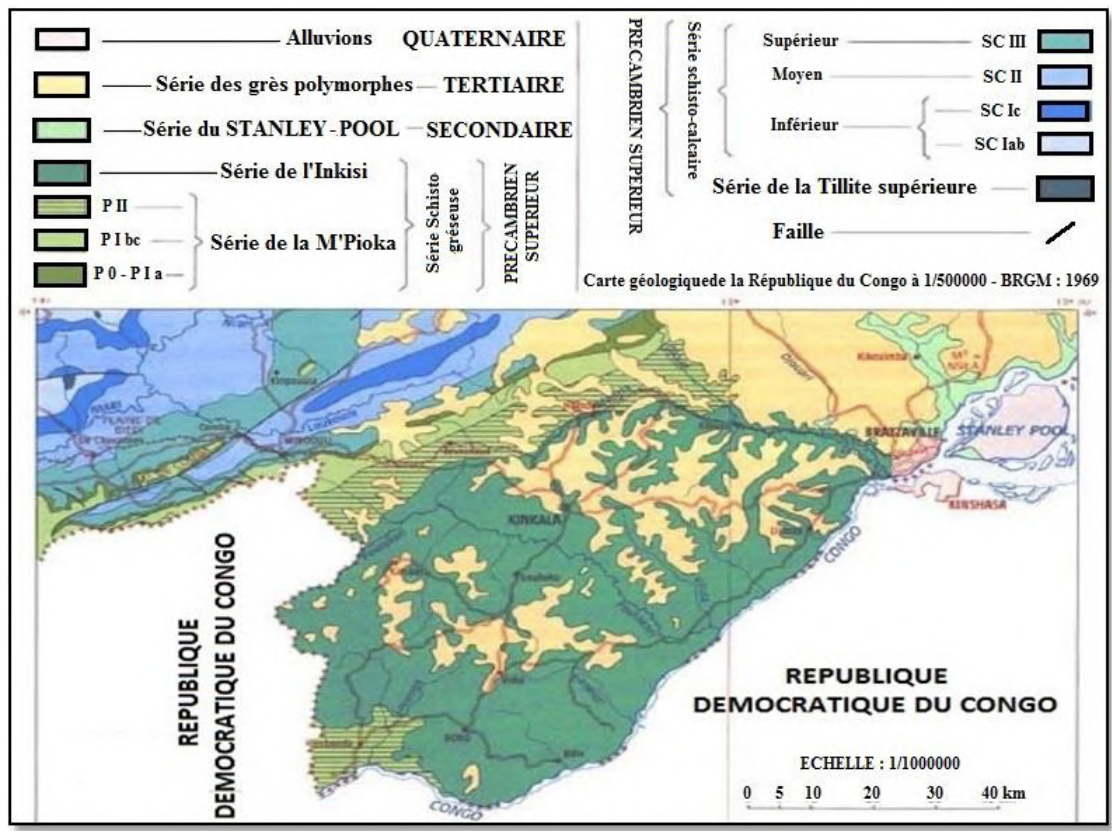

Figure 3. Carte géologique du Congo (Dadet, 1969).

- un niveau inférieur d'argilites rouges à passé gréseuse, d'âge jurassique supérieur. On y trouve des fossiles d'Estherie, d'Ostracodes et de Gastéropode. Ce niveau a été seulement rencontré en sondages à Brazzaville. Il correspondrait au remplissage fluvio-lacustre de certaines dépressions de la pénéplaine antérieure ;

- un niveau moyen de grès compacts blancs d'âge crétacé moyen qui est transgressif par rapport à la base, localement indurés et parfois lités sans stratifications entrecroisées. Il peut être rencontré à Brazzaville et près de Goma Tsé-tsé (Dadet, 1969) ;

- un niveau supérieur de grès kaoliniques tendres à grains moyens jaunâtres d'âge crétacé moyen et à stratifications entrecroisées nettes. Il s'agit de formations dunaires édifiées en milieu désertique. Ce niveau affleure dans l'île Mbamou, dans la vallée de Djili et sur les rives du Congo en amont de Brazzaville ;

- les caractères des grès kaoliniques du niveau supérieur les rapprocheraient beaucoup plus des grès polymorphes qu'à des grès moyens du Stanley-Pool qui correspondent à d'autres conditions de dépôt (Dadet, 1969).

La série des plateaux Batéké d'âge tertiaire et d'environ $400 \mathrm{~m}$ d'épaisseur (Moukolo et al., 1992; Desthieux et al., 1993), est essentiellement constituée de grès silicifiés et sables d'origine éolienne (Moukolo et al., 1992 ; Cosson, 1955). Cosson (1955) reconnait deux niveaux: le $\mathrm{Ba} 1$ et le $\mathrm{Ba} 2$ correspondant respectivement aux grès 
polymorphes d'âge paléogène et aux sables ocres d'âge néogène (Dadet, 1969). Les sables jaunes ocres du Bal constituent actuellement une formation à part entière appelée formation de couverture d'âge holocène récent (3000 ans à 2000 ans BP) (Thieblemont, 2013).

- les couches inférieures, des grès polymorphes d'âge éocène $(\mathrm{Ba} 1) \mathrm{de}$ 50 à $280 \mathrm{~m}$ d'épaisseur comprennent de bas en haut (Moukolo et al., 1992) :

- les grès tendres blancs-jaunâtres, gris par altération et grès rouges. Leur épaisseur est de quelques dizaines de mètres ;

- les grès durs silicifiés, quartziques clairs, blancs, bruns ou rosés pouvant passer latéralement à des roches silicifiées diverses : calcédoines, calcédoines gréseuses, grès à nodules calcédonieux avec de nombreuses micro géodes de quartz. On les qualifie de grès polymorphes à cause de leur multiple aspect ;

- les grès tendres, blancs qui se désagrègent en sables blancs.

- Les couches supérieures, des sables ocres ou limons sableux d'âge holocène récent, anciennement appelées (Ba2) de $90 \mathrm{~m}$ d'épaisseur. Elles reposent en discordance de ravinement sur l'ensemble des formations antérieures d'âge varié (Miyouna et al., 2016). Ces sables forment un ensemble d'apparence homogène par la granulométrie et la structure, cependant on peut distinguer deux zones qui correspondent à deux épisodes dans la sédimentation, en rapport avec la dynamique des vents (Miyouna et al., 2016). Ces couches constituent les parties hautes des plateaux tabulaires Koukouya, Djambala, Mbé et Nsa (AssiKaudjhis et al., 2010). Leur puissance diminue du SE au NW de 90 à 40 m (Dadet, 1969).

Les travaux de Serrane et al. (2008) ont montré dans les sables Batéké au Gabon (Ba1), l'existence de sources de zircons néoprotérozoïques. Ces sources sont distantes les unes des autres, ce qui prouve que les sables Batéké ont été érodés puis transportés par des processus éoliens.

Notons cependant que, la limite entre la série du Stanley-Pool et la série des plateaux Batéké n'a jamais été prouvée, car elle est ennoyée sous les sables provenant de la désagrégation des grès polymorphes qui constituent les plateaux, alors que le contact entre les sables ocres et les grès polymorphes est toujours net à cause de la différence des faciès et de la structure des terrains (Moukolo et al., 1992).

Moukolo et al. (1992) résume ainsi les grandes phases de l'évolution des plateaux Batéké de la manière suivante :

- le dépôt de la série du Stanley-Pool s'est fait dans un milieu fluciolacustre. Ce milieu devient aride, ce qui se traduit par une silicification ; 
- le dépôt des grès Batéké s’est fait dans un environnement éolien aride couramment interrompu par des phases lacustres.

\subsubsection{Contexte hydrogéologique}

Le contexte hydrogéologique des plateaux Batéké présente une structure d'ensemble assez simple, avec les aquifères non confinés encore appelés nappes libres constitués principalement d'alluvions quaternaires, de sables et grès tendres des séries Batéké et des grès continentaux de la série du Stanley-Pool (Moukolo et al., 1992). Ce sont des aquifères continus des bassins sédimentaires d'âge mésozoïque à quaternaire constitués des limons sableux d'âge néogène et des grès tendres d'âge paléogène. Moukolo et al. (1992) reconnaissent dans les plateaux Batéké, des terrains perméables et des terrains très peu perméables :

- les terrains reconnus comme perméables: dans la région de Brazzaville, plusieurs mesures de perméabilité ont été effectuées sur des sondages de recherches ou des ouvrages d'exploitation des eaux souterraines. Les coefficients moyens de Darcy obtenus sont compris entre $10^{-4} \mathrm{~m} / \mathrm{s}$ pour les anciens alluvions du fleuve Congo et $10^{-7} \mathrm{~m} / \mathrm{s}$ pour les limons sableux du $\mathrm{Ba} 2$ (formation de couverture). Les niveaux caillouteux alluviaux sont très circonscrits, leur épaisseur est très faible et très irrégulière. Ces dépôts ne présentent que peu d'intérêt hydrogéologique (Moukolo et al., 1992).

Les grès tendres et les sables du Bal et du Stanley-Pool ont une extension beaucoup plus grande et leur épaisseur peu atteindre plusieurs centaines de mètres. Ces terrains constituent malgré leurs coefficients de Darcy assez faibles $\left(10^{-5} \mathrm{~m} / \mathrm{s}\right)$, les niveaux les plus intéressants du point de vue hydrogéologique (Moukolo et al., 1992).

- les terrains considérés comme très peu perméables, on a :

- la fraction argileuse des grès et limons sableux : on note une prédominance de kaolinite dans les grès polymorphes et les grès du Stanley-Pool. Ces sédiments présentent un grand intérêt hydrogéologique à cause de leur forte capacité de rétention (Moukolo, 1992) ;

- les silicifications : les silicifications en calcédoine, en forme de dalle discontinue s'observent dans des régions argileux kaoliniques des bas-fonds dans la région de Brazzaville. Leur épaisseur est très faible, moins d'un mètre. On y trouve des grès calcédonieux, situés au-dessus du SP2 de nombreuses sources de contact (Moukolo, 1992).

D'un point de vue hydrogéologique du plateau de Mbé, les travaux de Moukolo et al. (1992) ont été significatifs pour la connaissance de son potentiel hydrogéologique. Selon Moukolo (1992), il existe une nappe libre 
profonde bombée sur ce plateau. Comme le plateau est à la fois plus grand et moins élevé, le sommet de la nappe est beaucoup plus proche de la surface topographique à tel point que cette dernière recoupe la surface de la nappe au niveau des deux vallées de la Mary et de la Gamboma.

\subsection{Méthodologie utilisée}

\subsubsection{Acquisition des données hydrogéologiques}

Dans le cadre cette étude, une campagne d'échantillonnage des eaux souterraines et de surface a été réalisée en juillet 2013 dans le plateau de Mbé (Figure 4).

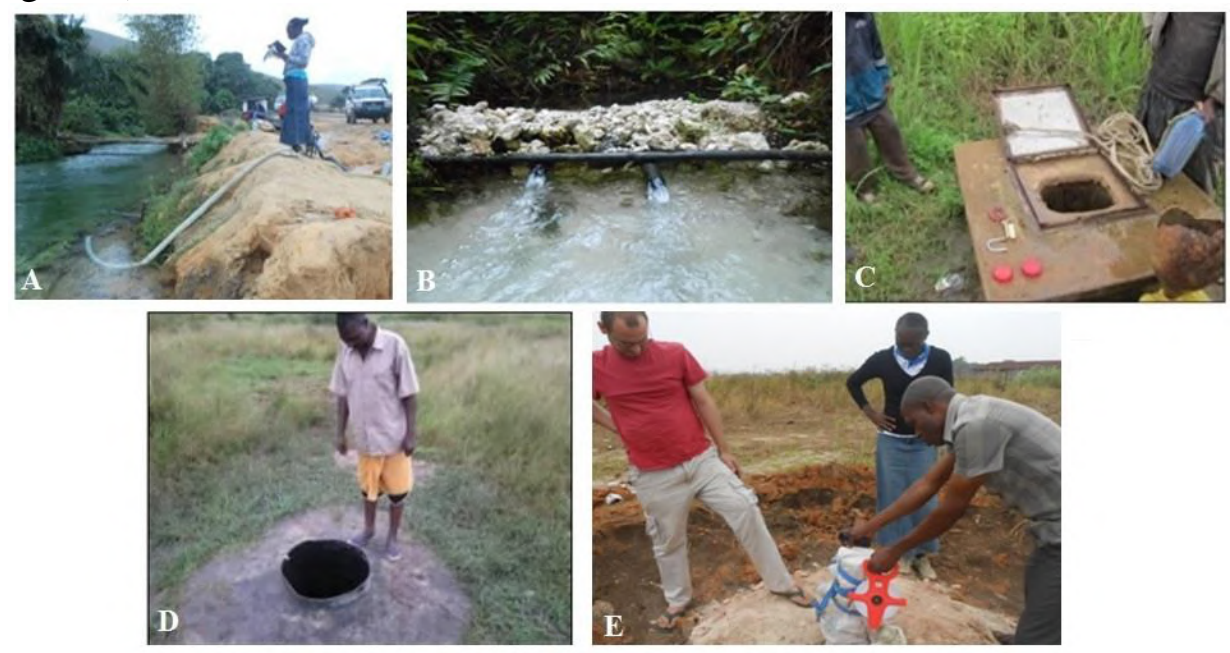

Figure 4. Eaux de surface (Photo A), eaux de source (Photo B).

Puits traditionnels (Photos Cet D) et forage de reconnaissance (Photo E).

Au cours de cette campagne d'échantillonnage, 56 points d'eau ont été prélevés dont 9 eaux de surface, 10 sources, 22 puits traditionnels et 16 forages. Ces échantillons d'eau ont été recueillis dans des bouteilles en polyéthylène de capacité $1,5 \mathrm{~L}$ en vue des analyses physico-chimiques au laboratoire de contrôle et d'analyses des eaux de la Société Nationale de Distribution des Eaux (SNDE) aujourd'hui La Congolaise des Eaux (LCDE). Au laboratoire de La Congolaise des Eaux (LCDE) les analyses chimiques ont porté sur les éléments majeurs et mineurs suivants : $\mathrm{Cl}^{-}, \mathrm{SO}_{4}{ }^{2-}, \mathrm{NO}_{3}{ }^{-}, \mathrm{HCO}_{3}{ }^{-}$, $\mathrm{PO}_{4}{ }^{3-}, \mathrm{F}^{-}, \mathrm{Na}^{+}, \mathrm{K}^{+}, \mathrm{Mg}^{2+}, \mathrm{Ca}^{2+}, \mathrm{Al}^{3+}, \mathrm{Pb}^{2+}, \mathrm{Fe}^{2+}, \mathrm{Mn}^{2+}$. Le $\mathrm{pH}$ et la conductivité électrique ont été déterminés sur le terrain à partir d'un $\mathrm{pH}$-mètre et d'un conductimètre portatifs.

La Figure 5 représente la carte de la localisation des différents points d'eau échantillonnés dans le plateau de Mbé en juillet 2013. 


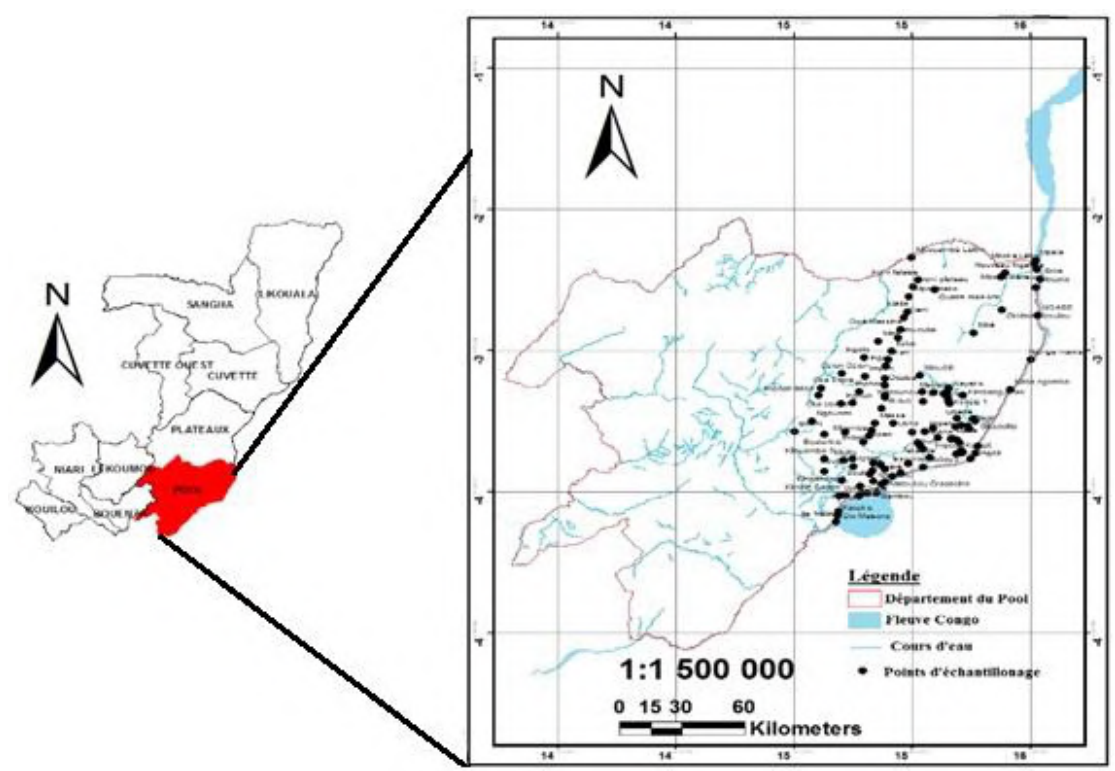

Figure 5. Localisation des points de prélèvements (Sabata, 2017).

\subsubsection{Le sondage électrique vertical}

La méthode mise en œuvre dans le cadre de cette étude est le sondage électrique avec le dispositif Schlumberger. Cette méthode a été choisie pour trois raisons essentielles : elle est simple à manipuler, économique et les contrastes de résistivité entre les différents terrains sont suffisants. Le principe du sondage électrique consiste à établir la courbe de variation de la résistivité apparente du sous-sol en fonction de la distance de la ligne de courant $\mathrm{AB}$ grâce au dispositif quadripôle AMNB en surface.

$\mathrm{Au}$ cours de cette campagne de prospection géophysique, onze (11) sondages électriques verticaux $(\mathrm{AB} / 2=500 \mathrm{~m})$ ont été réalisés dont cinq $(05)$ sondages sur le site du village Imvouba, cinq (05) à Ingah et un sondage de calage de résistivités des formations géologiques au niveau du forage du nouveau village agricole d'Imvouba où nous disposons des données techniques (Figure 6). 


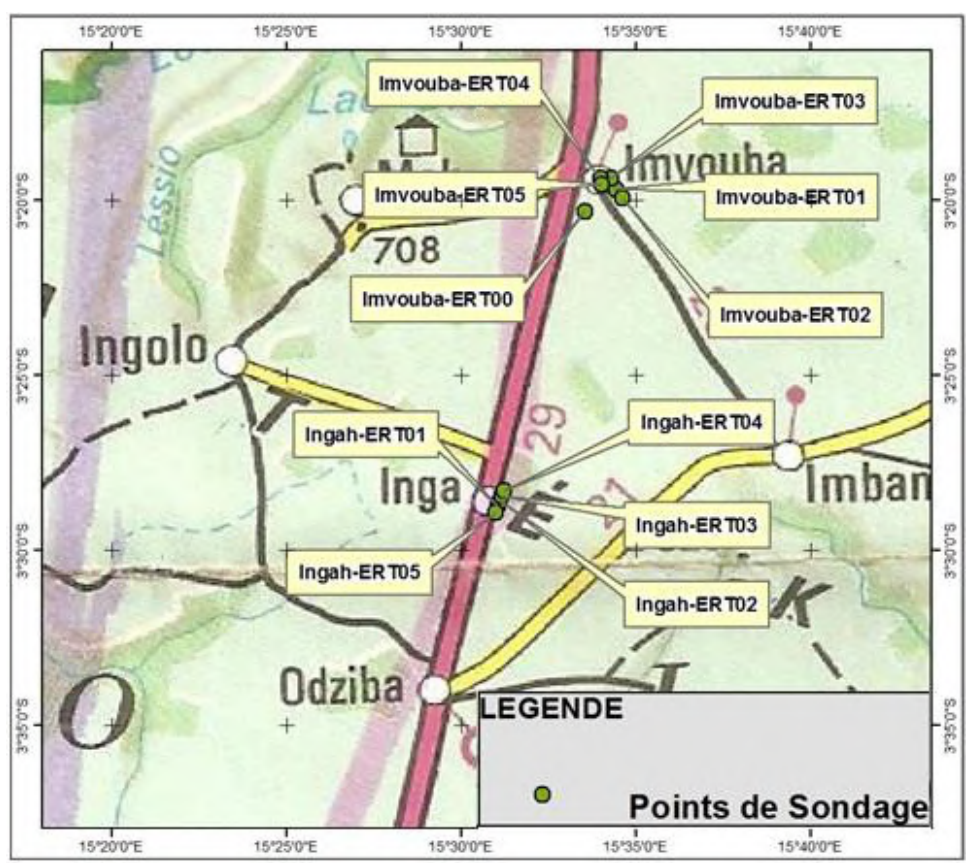

Figure 6. Localisation des sondages électriques verticaux réalisés (Fouroux, 2015).

Les longueurs des lignes $\mathrm{AB} / 2$ varient entre 450 et $500 \mathrm{~m}$. Ces longueurs de lignes ont été choisies pour prospecter à de très grandes profondeurs (Figure 7). Pour s'assurer de la fiabilité des mesures sur le terrain, nous avons pris des dispositions pratiques visant à vérifier systématiquement les valeurs de résistance de prise au sol au niveau des électrodes avant de faire les mesures. Toutefois, si les valeurs de prise des électrodes sont élevées (audelà de $10 \mathrm{ohm} . \mathrm{m})$, les électrodes d'injection et de réception sont arrosées avec de l'eau salée jusqu'à ce que celles-ci soient en-dessous de la valeur de 10 ohm.m. 


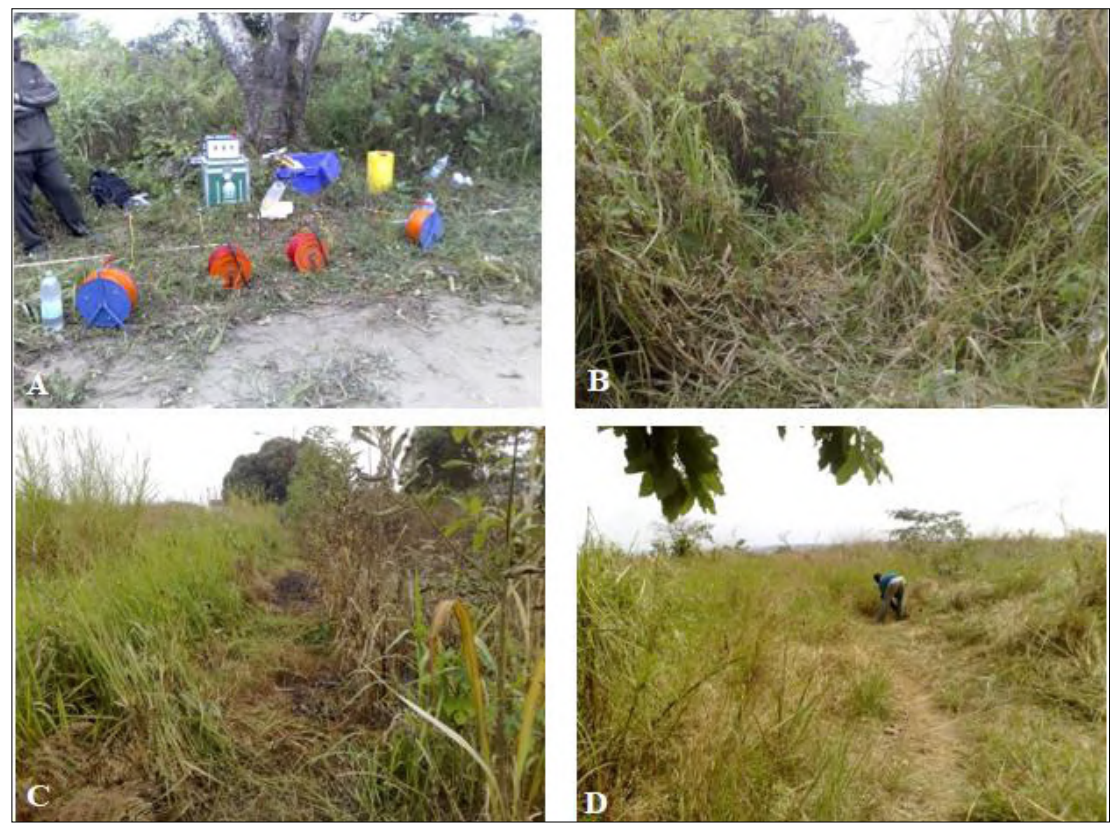

Figure 7. Résistivimètre de type Syscal pro Switch en station (Photo A) et layons pour les lignes de sondages électriques verticaux (Photos B, C et D) (Fouroux, 2015).

\subsubsection{Travaux de foration}

La méthode utilisée pour la réalisation des forages est le rotary à la boue. Le rotary est utilisé dans les terrains sédimentaires non consolidés. Pour les puissantes machines de forage (cas des appareils pétroliers), elle peut être utilisée en terrains durs. Un outil appelé tricône ou trillâmes est mis en rotation depuis la surface du sol par l'intermédiaire d'un train de tige. L'avancement de l'outil s'effectue par abrasion du terrain, sans choc, uniquement par rotation et poussée. Celle-ci est fournie par la puissance de la machine mais surtout par le poids des tiges au-dessus de l'outil. Certaines tiges spécifiques sont réservées à cet usage : ce sont des masses tiges. En ce qui nous concerne, nous n'avons pas eu le besoin de les utiliser.

\section{Resultats et Interpretations}

\subsection{Résultats hydrogéologiques}

Dans le Plateau de Mbé, une première description physico-chimique complète des eaux souterraines et de surface a été faite par Sabata en 2017. Cette description avait montré que les eaux souterraines et de surface du Plateau de Mbé sont acides, douces, faiblement minéralisées et de faciès chimique dominé par les bicarbonatés calciques. 


\subsubsection{Caractéristiques physico-chimiques des eaux}

Le Tableau 1 ci-dessous, présente les paramètres statistiques (minimum, maximum, moyenne, écart type $(\mathrm{S})$ et coefficient de variation $(\mathrm{CV})$ ) calculés pour chacune des variables physiques et chimiques des eaux souterraines et de surface de la campagne de juillet 2013.

Tableau 1. Paramètres statistiques des paramètres physico-chimiques des eaux (Juillet 2013).

\begin{tabular}{|c|c|c|c|c|c|c|c|c|c|c|}
\hline \multirow{2}{*}{$\begin{array}{l}\text { Paramètres physico- } \\
\text { chimiques }\end{array}$} & \multicolumn{5}{|c|}{ Eaux souterraines } & \multicolumn{5}{|c|}{ Eaux de surface } \\
\hline & Min. & Max. & Moy. & $\mathrm{S}$ & $\mathrm{CV}$ & Min. & Max. & Moy. & $\mathrm{S}$ & $\mathrm{CV}$ \\
\hline pH & 4,85 & 7,37 & 6,04 & 0,53 & 8,80 & 5,47 & 6,76 & 5,99 & 0,39 & 6,59 \\
\hline C.E $(\mu \mathrm{s} / \mathrm{cm})$ & 0,01 & 105,70 & 22,49 & 21,09 & 93,77 & 3,64 & 34,60 & 10,76 & 9,32 & 86,62 \\
\hline T.D.S (mg/L) & 2,10 & 47,61 & 10,45 & 9,49 & 90,75 & 1,75 & 15,58 & 4,87 & 4,17 & 85,74 \\
\hline $\mathrm{Ca}^{2+}(\mathrm{mg} / \mathrm{L})$ & 0,70 & 20,24 & 4,04 & 4,22 & 104,62 & 0,01 & 8,20 & 1,86 & 2,45 & 131,76 \\
\hline $\mathrm{Mg}^{2+}(\mathrm{mg} / \mathrm{L})$ & 0,02 & 2,37 & 0,62 & 0,68 & 109,77 & 0,02 & 1,20 & 0,43 & 0,37 & 86,67 \\
\hline $\mathrm{Na}^{+}(\mathrm{mg} / \mathrm{L})$ & 0,08 & 4,23 & 0,72 & 0,75 & 105,23 & 0,08 & 0,89 & 0,31 & 0,24 & 77,34 \\
\hline $\mathrm{K}^{+}(\mathbf{m g} / \mathbf{L})$ & 0,00 & 2,76 & 0,47 & 0,51 & 108,17 & 0,04 & 0,61 & 0,23 & 0,19 & 84,15 \\
\hline $\mathrm{HCO}_{3}^{-}(\mathrm{mg} / \mathrm{L})$ & 0,00 & 25,76 & 7,33 & 5,49 & 74,84 & 1,61 & 25,76 & 6,45 & 7,43 & 115,32 \\
\hline $\mathrm{Cl}^{-}(\mathrm{mg} / \mathrm{L})$ & 0,00 & 9,17 & 2,66 & 2,40 & 90,19 & 0,21 & 3,64 & 1,15 & 0,99 & 86,22 \\
\hline $\mathrm{SO}_{4}{ }^{2-}(\mathrm{mg} / \mathrm{L})$ & 0,00 & 2,86 & 0,21 & 0,50 & 232,30 & 0,00 & 0,65 & 0,10 & 0,21 & 217,84 \\
\hline $\mathrm{NO}_{3}^{-}(\mathrm{mg} / \mathrm{L})$ & 0,06 & 57,72 & 6,29 & 10,80 & 171,83 & 0,04 & 2,18 & 0,63 & 0,66 & 106,05 \\
\hline $\mathrm{Fe}^{2+}(\mathrm{mg} / \mathrm{L})$ & 0,00 & 1,09 & 0,06 & 0,17 & 298,13 & 0,00 & 0,35 & 0,10 & 0,12 & 119,57 \\
\hline $\mathrm{Mn}^{2+}(\mathbf{m g} / \mathbf{L})$ & 0,00 & 4,80 & 0,50 & 0,89 & 177,25 & 0,00 & 0,03 & 0,01 & 0,01 & 202,19 \\
\hline $\mathrm{PO}_{4}{ }^{3-}(\mathrm{mg} / \mathrm{L})$ & 0,00 & 0,31 & 0,02 & 0,06 & 259,37 & 0,00 & 1,23 & 0,44 & 0,51 & 115,70 \\
\hline $\mathrm{Al}^{3+}(\mathbf{m g} / \mathbf{L})$ & 0,00 & 4,44 & 0,13 & 0,64 & 490,77 & 0,00 & 0,04 & 0,02 & 0,01 & 87,38 \\
\hline
\end{tabular}

Le coefficient de variation est un paramètre de mesure de la dispersion d'une série d'observations d'une variable autour de sa moyenne arithmétique. Si la valeur du CV est inférieure à $50 \%$, la variable observée a une distribution homogène autour de sa moyenne arithmétique et si la valeur du CV est supérieure ou égale à $50 \%$, la variable observée a une distribution hétérogène autour de sa moyenne arithmétique.

En juillet 2013, les CV différents paramètres physico-chimiques calculés, montrent que seules les valeurs de $\mathrm{pH}$ ont une distribution géographique homogène $(\mathrm{CV}<50 \%)$ aussi bien pour les eaux souterraines que les eaux de surface. D'où les paramètres physico-chimiques (CE, TDS, $\mathrm{Ca}^{2+}, \mathrm{Mg}^{2+}, \mathrm{Na}^{+}, \mathrm{K}^{+}, \mathrm{Fe}^{2+}, \mathrm{Mn}^{2+}, \mathrm{Al}^{3+}, \mathrm{HCO}_{3}{ }^{-}, \mathrm{NO}_{3}{ }^{-}, \mathrm{Cl}^{-}, \mathrm{SO}_{4}{ }^{2-}$ et $\left.\mathrm{PO}_{4}{ }^{3-}\right)$ ayant un coefficient de variation hétérogène $(\mathrm{CV}>50 \%)$ permettent de dire que les eaux souterraines et de surface de juillet 2013 présentent de fortes différences de qualité physico-chimique d'un point à l'autre dans le secteur du plateau de Mbé.

La minéralisation d'une eau peut être évaluée par sa conductivité électrique ou par le taux de solides dissous (TDS). En fonction des valeurs de la conductivité électrique, les eaux peuvent être classées en cinq (5) classes d'après Rodier (1996) : $\mathbf{C E}<\mathbf{1 0 0} \boldsymbol{\mu S} / \mathbf{c m}$, eau de minéralisation très faible ; 
$100<\mathrm{CE}<200 \mu \mathrm{S} / \mathrm{cm}$, eau de minéralisation faible $; 200<\mathrm{CE}<333 \mu \mathrm{S} / \mathrm{cm}$, eau de minéralisation moyenne $; 333<\mathbf{C E}<666 \mu \mathrm{S} / \mathbf{c m}$, eau de minéralisation accentuée ; $666<\mathbf{C E}<1000 \mu \mathrm{S} / \mathbf{c m}$, eau de minéralisation importante ; CE $>1000 \mu \mathrm{S} / \mathbf{c m}$, eau de minéralisation élevée.

Les valeurs de la conductivité électrique mesurées en juillet 2013 varient de $0,01 \mu \mathrm{S} / \mathrm{cm}($ Pts-21) à $105,70 \mu \mathrm{S} / \mathrm{cm}(\mathrm{F}-08)$ pour les eaux souterraines et de $3,64 \mu \mathrm{S} / \mathrm{cm}$ (ES-06) à $34,60 \mu \mathrm{S} / \mathrm{cm}$ (ES-04) pour les eaux de surface (Sabata, 2017). Les eaux souterraines du Plateau de Mbé sont dans l'ensemble de minéralisation très faible. Tandis qu'en fonction des valeurs du TDS, les eaux peuvent être classées en quatre (4) classes d'après Banton et al. (1997) : les eaux douces (TDS < $\mathbf{1 0 0 0} \mathbf{~ m g / L )}$; les eaux saumâtres $(\mathbf{1 0 0 0} \mathbf{~ m g / L}$ $<$ TDS $<10000 \mathrm{mg} / \mathrm{L})$; les eaux salines $(10000 \mathrm{mg} / \mathrm{L}<\mathrm{TDS}<100000 \mathrm{mg} / \mathrm{L})$ et les saumures $($ TDS $>\mathbf{1 0 0 0 0 0 ~} \mathbf{~ m g} / \mathbf{L}$ ).

Ainsi, suivant les différentes classes établies par Banton et al. (1997), les eaux souterraines et de surface sont douces car leurs TDS sont compris entre $2,10 \mathrm{mg} / \mathrm{L}(\mathrm{F}-11)$ et $47,61 \mathrm{mg} / \mathrm{L}(\mathrm{F}-08)$ pour les eaux souterraines et entre $1,75 \mathrm{mg} / \mathrm{L}$ (ES-06) et $15,58 \mathrm{mg} / \mathrm{L}$ (ES-04) pour les eaux de surface (Sabata, 2017).

Les valeurs de $\mathrm{pH}$ varient de 4,85 (F-08) à 7,37 (F-16) pour les eaux souterraines et entre 5,47 (ES-09) et 6,76 (ES-04) pour les eaux de surface. Ces valeurs de $\mathrm{pH}$ indiquent que les eaux souterraines et de surface sont acides à légèrement neutres et que l'alcalinité des eaux serait contrôlée essentiellement par les ions bicarbonates $\left(\mathrm{HCO}_{3}{ }^{-}\right)$.

Pour déterminer les familles et faciès chimiques des eaux souterraines et de surface, les données des analyses chimiques de la campagne de juillet 2013 ont été reportées sur le diagramme triangulaire de Piper (Figure 8).

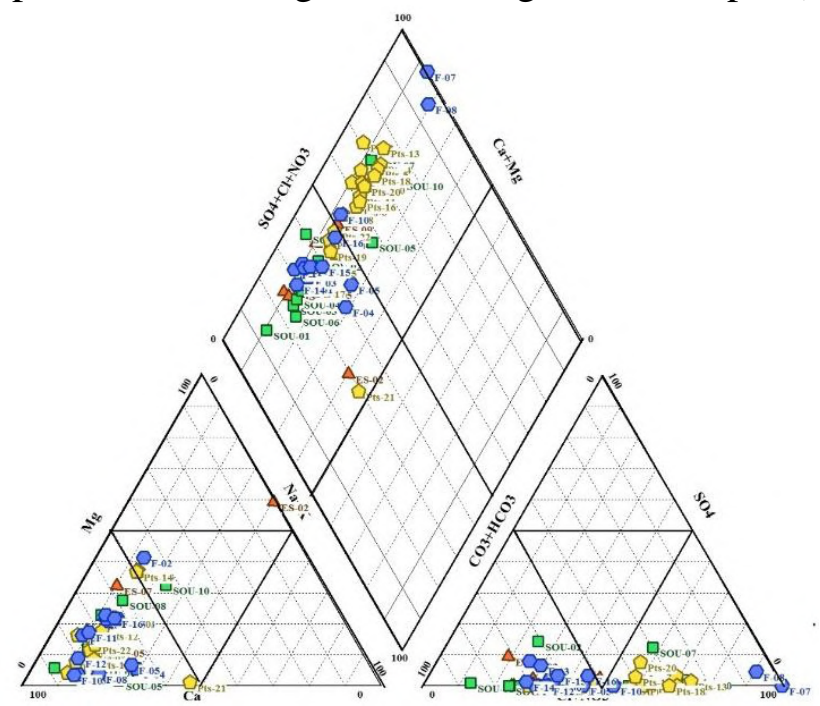

Figure 7. Diagramme de Piper des eaux souterraines et de surface (juillet 2013). 
Le diagramme losangique de Piper montre que les eaux souterraines et de surface du plateau de Mbé sont caractérisées par deux familles chimiques, à savoir : les eaux chlorurées sulfatées calciques et magnésiennes qui représente $44 \%$ et les eaux bicarbonatées calciques et magnésiennes qui représente $56 \%$.

Cependant dans le diagramme triangulaire des cations, les points d'eaux se répartissent en trois groupes : le premier à évolution vers le pôle calcique (plus de 90 \%), le deuxième à évolution vers la zone mixte (moins de $10 \%$ ) et enfin vers le pôle magnésien (ES-02).

Dans le diagramme triangulaire des anions, on distingue deux groupes de points : le premier à évolution vers le pôle bicarbonaté (plus de $70 \%$ ) et le deuxième à évolution vers le pôle chloruré (moins de $30 \%$ ).

Le report des analyses chimiques des eaux souterraines et de surface de la campagne de juillet 2013 sur le diagramme de Schöeller-Berkaloff, montre des eaux dominées par le faciès chimique bicarbonaté calcique (Figure 9).
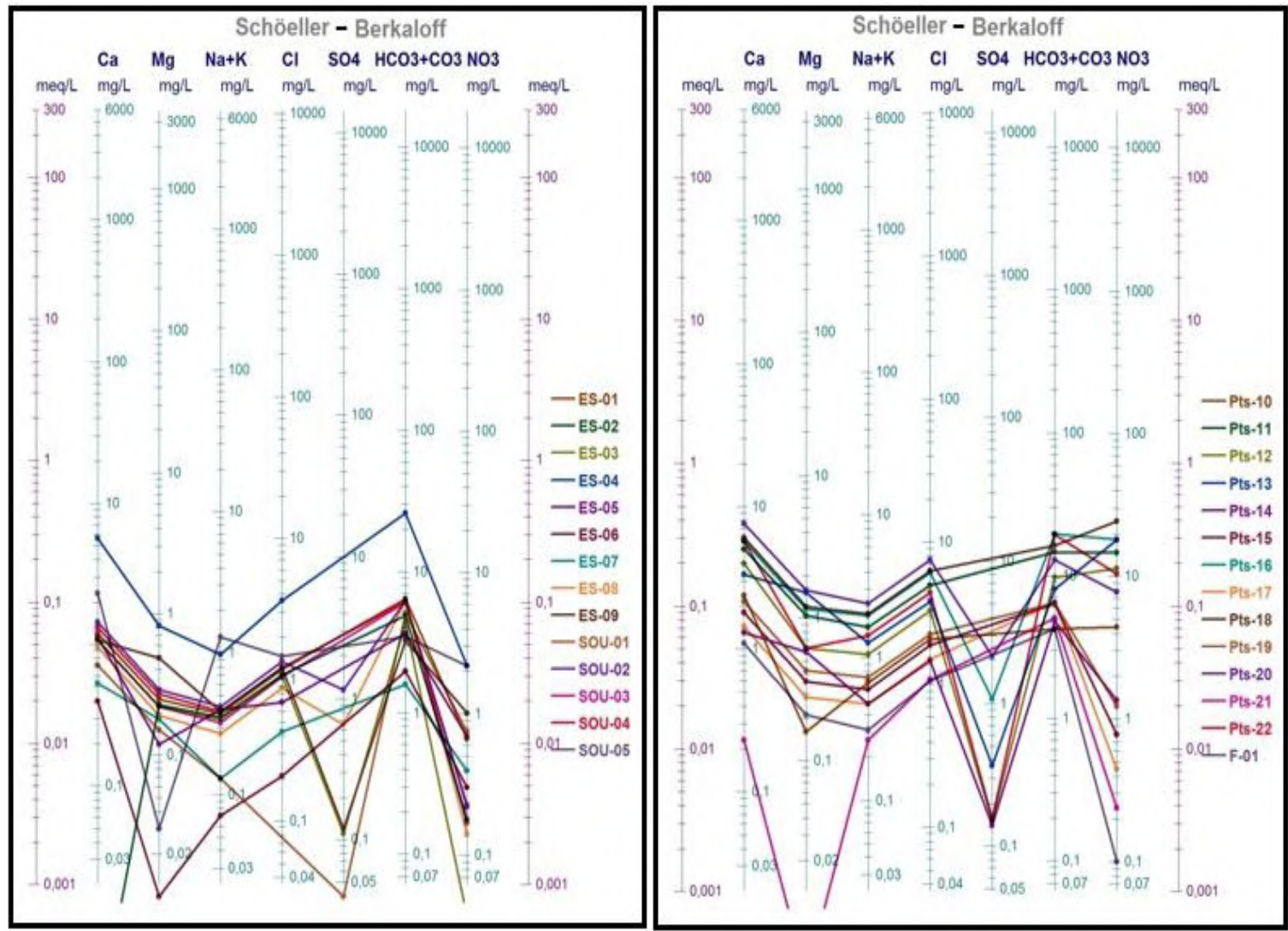

Figure 9a. Diagramme de Schöeller-Berkaloff des eaux de surface, de sources et de puits traditionnels (juillet 2013) 

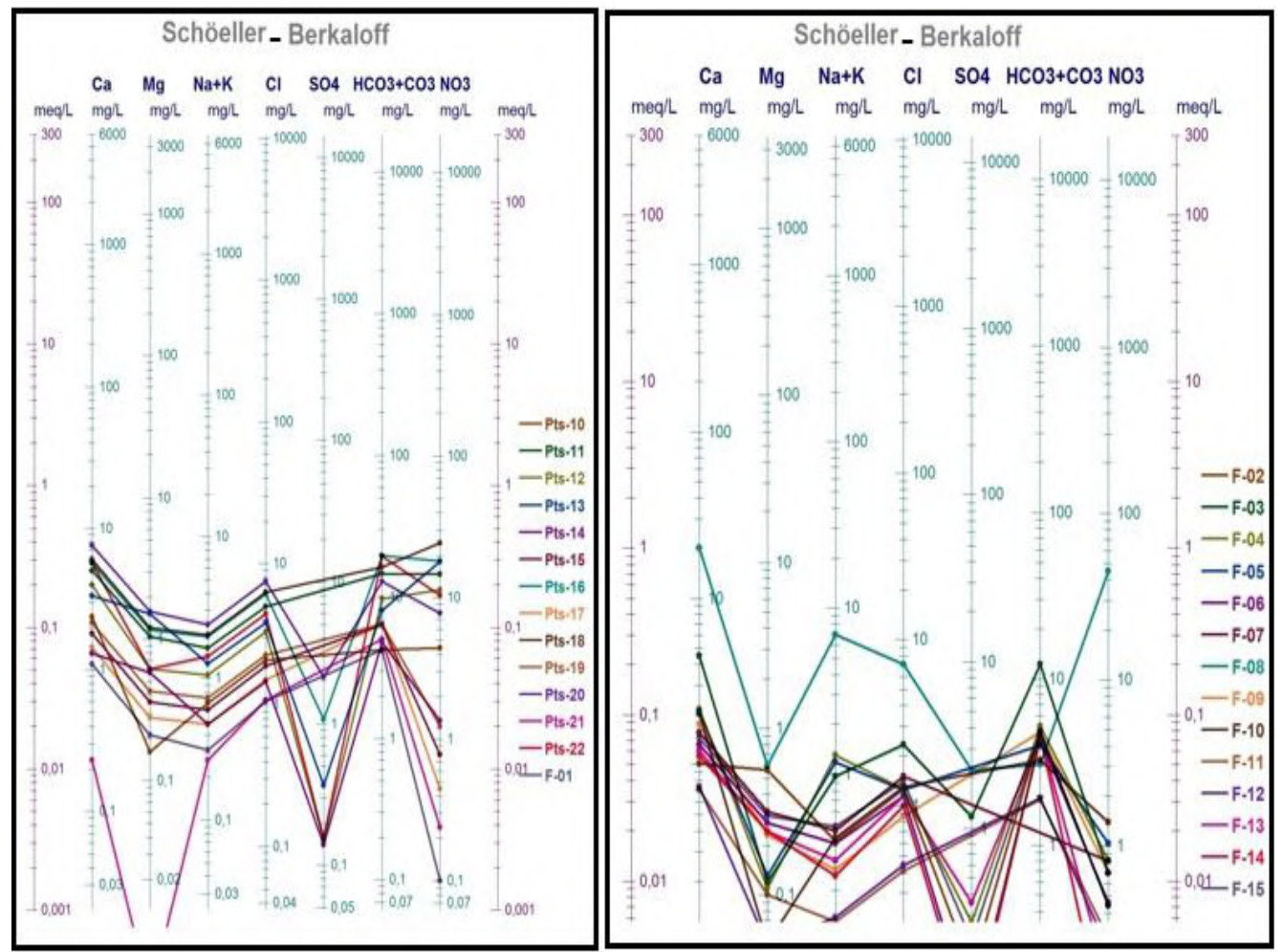

Figure 9b. Diagramme de Schöeller-Berkaloff des eaux de puits traditionnels et des forages (juillet 2013)

\subsubsection{Qualité physico chimique des eaux souterraines et de surface}

Dans le cadre de cette étude, nous nous limiterons à l'évaluation de la potabilité de l'eau de boisson qui est fondée sur la comparaison entre les résultats de l'analyse qualitative de l'eau et les valeurs guides de l'OMS requises pour une eau de boisson. Ainsi, le Tableau 2 ci-après met en évidence les normes de potabilité de l'OMS (2017) et les teneurs des paramètres physico-chimiques (minimum et maximum) des eaux souterraines et de surface (juillet 2013).

Le problème majeur de la qualité de l'eau destinée à la consommation humaine a été longtemps d'ordre sanitaire. Par conséquent, l'analyse comparative des paramètres physico-chimiques des eaux souterraines et de surface du plateau de Mbé (juillet 2013) avec les normes admissibles définies par l'OMS (2017) pour l'eau de boisson, a permis de savoir que la bonne qualité physico-chimique des eaux souterraines et de surface est remise en cause par la présence de certains éléments comme le fer total $\left(\mathrm{Fe}^{2+}+\mathrm{Fe}^{3+}\right)$, le manganèse $\left(\mathrm{Mn}^{2+}\right)$, les phosphates $\left(\mathrm{PO}_{4}^{3-}\right)$ et l'aluminium $\left(\mathrm{Al}^{3+}\right)$ à des teneurs élevées dépassant parfois les concentrations maximales admissibles pour l'eau de boisson définies par l'OMS. 
Tableau 2. Normes de potabilité de l'OMS (2017) et teneurs des paramètres physicochimiques des eaux (Sabata, 2013).

\begin{tabular}{|c|c|c|c|c|c|}
\hline \multirow{3}{*}{$\begin{array}{c}\text { Paramètres } \\
\text { physico-chimiques }\end{array}$} & \multirow{3}{*}{$\begin{array}{c}\text { Valeurs } \\
\text { maximales } \\
\text { admissibles } \\
\text { par l'OMS } \\
(2017)\end{array}$} & \multicolumn{4}{|c|}{ Campagne de juillet 2013} \\
\hline & & \multicolumn{2}{|c|}{ Eaux souterraines } & \multicolumn{2}{|c|}{ Eaux de surface } \\
\hline & & Minimum & Maximum & Minimum & Maximum \\
\hline $\mathrm{pH}$ & 8,5 & 5,27 & 7,23 & 5,47 & 6,76 \\
\hline $\mathrm{CE}(\mu \mathrm{s} / \mathrm{cm})$ & 2000 & 0,01 & 63,00 & 3,64 & 34,60 \\
\hline TDS & 600 & 2,70 & 28,37 & 1,75 & 15,58 \\
\hline $\mathrm{Ca}^{2+}(\mathrm{mg} / \mathrm{L})$ & 100 & 0,23 & 11,79 & 0,00 & 5,60 \\
\hline $\mathrm{Mg}^{2+}(\mathrm{mg} / \mathrm{L})$ & 50 & 0,00 & 1,57 & 0,01 & 0,82 \\
\hline $\mathbf{K}^{+}(\mathrm{mg} / \mathbf{L})$ & 12 & 0,00 & 0,92 & 0,02 & 0,42 \\
\hline $\mathrm{Na}^{+}(\mathrm{mg} / \mathrm{L})$ & 100 & 0,10 & 1,48 & 0,05 & 0,56 \\
\hline $\mathrm{HCO}_{3}^{-}(\mathrm{mg} / \mathrm{L})$ & - & 1,83 & 19,52 & 1,61 & 25,76 \\
\hline $\mathrm{NO}_{3}^{-}(\mathrm{mg} / \mathrm{L})$ & 50 & 0,09 & 24,52 & 0,04 & 2,18 \\
\hline $\mathrm{Cl}(\mathrm{mg} / \mathrm{L})$ & 200 & 0,00 & 7,41 & 0,21 & 3,64 \\
\hline $\mathrm{SO}_{4}{ }^{2-}(\mathrm{mg} / \mathrm{L})$ & 250 & 0,00 & 2,12 & 0,00 & 0,65 \\
\hline Fe $\quad(\mathrm{mg} / \mathrm{L})$ & 0,3 & 0,00 & 1,09 & 0,00 & 0,35 \\
\hline $\mathrm{Mn}^{2+}(\mathrm{mg} / \mathrm{L})$ & 0,1 & 0,00 & 4,80 & 0,00 & 0,03 \\
\hline $\mathrm{PO}_{4}{ }^{3-}(\mathrm{mg} / \mathrm{L})$ & 0,5 & 0,00 & 0,31 & 0,00 & 1,23 \\
\hline $\mathrm{Al}^{3+}(\mathrm{mg} / \mathrm{L})$ & 0,2 & 0,00 & 4,44 & 0,00 & 0,04 \\
\hline
\end{tabular}

\subsection{Résultats des sondages électriques verticaux}

\subsubsection{Site du village Imvouba}

L'interprétation des courbes des sondages électriques verticaux (Figures 10 et 11) laisse apparaitre sur le site du village Imvouba cinq (5) types de couches de terrain aux résistivités apparentes différentes :

- une couche sommitale représentant le sol non différencié (sables limoneux) et sa partie végétale. Les résistivités observées varient de 580 à 2000 ohm.m et son épaisseur ne dépasse pas un mètre ; 

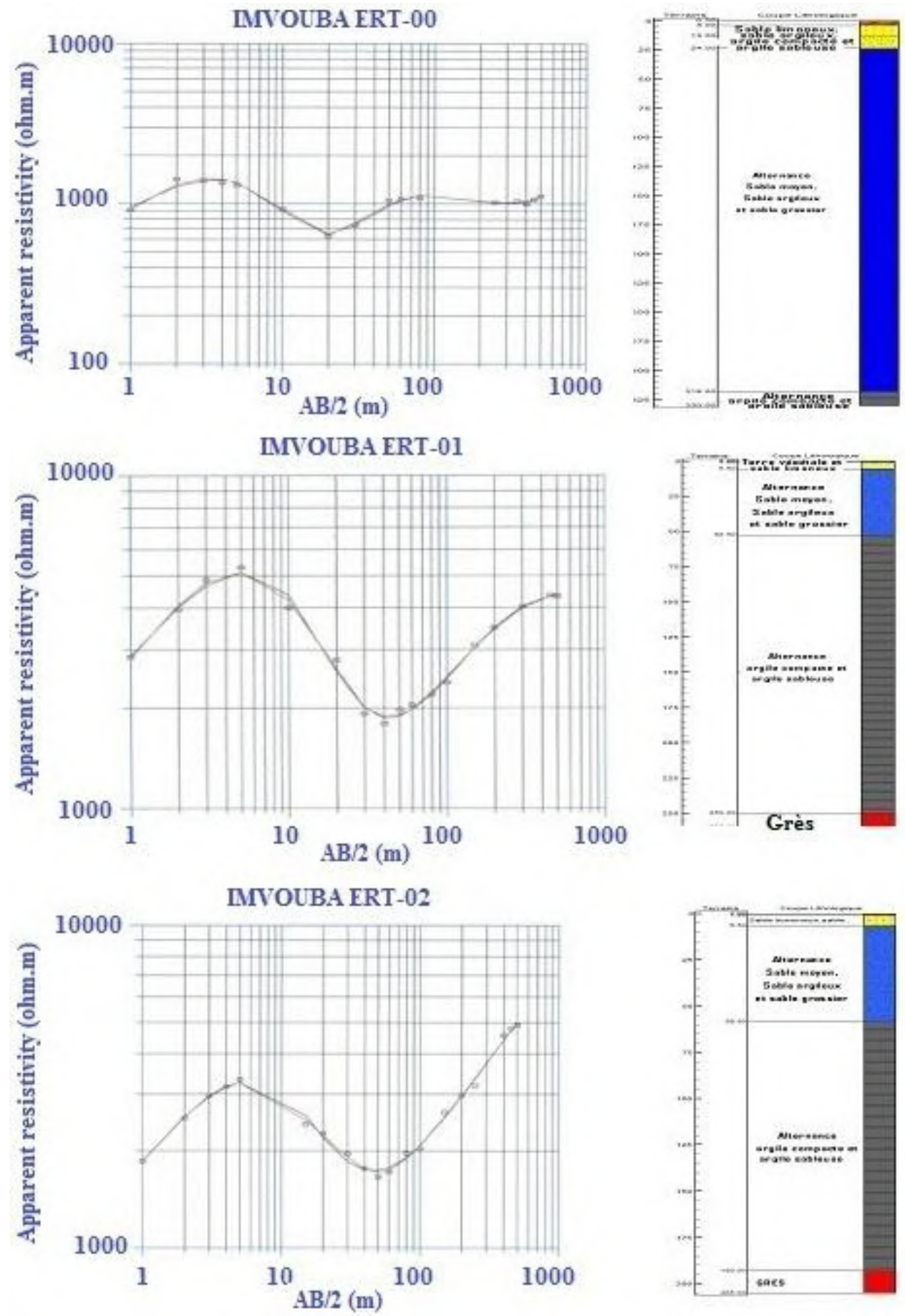

Figure 10. Variation de la résistivité en fonction de $\mathrm{AB} / 2$ au site du village Imvouba et modèle de sols associés (Fouroux, 2015). 

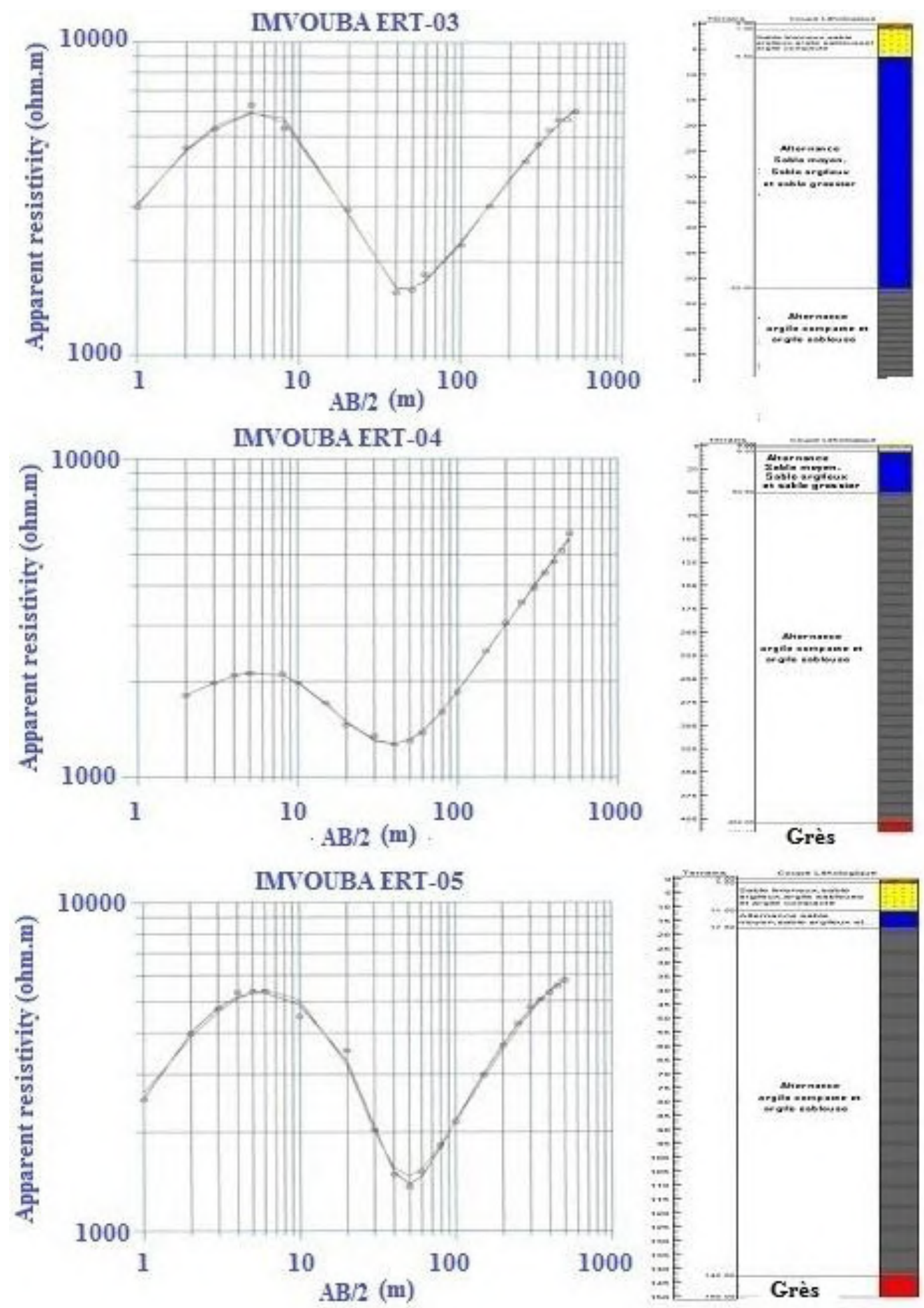

Figure 11. Variation de la résistivité en fonction de $A B / 2$ au site du village Imvouba et modèle de sols associés (Fouroux, 2015). 
- une deuxième couche représentant des sables limoneux, des sables argileux, des argiles sableuses et des argiles compactes avec des résistivités allant de 2400 à $7500 \mathrm{ohm}$.m. Son épaisseur varie de 5 à 20 $\mathrm{m}$;

- une troisième couche représentant l'alternance entre des sables moyens, des sables argileux et des sables grossiers. Cette couche caractérisée par de faibles résistivités $(150 \mathrm{ohm} . \mathrm{m}$ à $1650 \mathrm{ohm} . \mathrm{m})$ constitue le niveau aquifère. Son épaisseur varie de 6 à $53 \mathrm{~m}$;

- une quatrième couche formée par l'alternance entre argiles compactes et les argiles sableuses dont les résistivités sont comprises entre 4000 ohm.m et 15500 ohm.m. Son épaisseur varie de 120 à 353 m ;

- enfin une dernière couche avec une épaisseur infinie et des résistivités oscillant entre $5000 \mathrm{ohm}$.m et $54000 \mathrm{ohm} . \mathrm{m}$. Elle est représentée par les grès.

\subsubsection{Site du village Ingah}

Sur le site du village Ingah, l'interprétation des courbes des sondages électriques verticaux (Figures 12 et 13) laisse également apparaitre cinq (5) types de couches de terrains aux résistivités apparentes variables :

- une couche sommitale représentée par le sol non différencié (sable limoneux) et sa partie végétale avec des résistivités oscillant entre 50 et $2600 \mathrm{ohm} . \mathrm{m}$. Son épaisseur est comprise entre 3 et $5 \mathrm{~m}$;

- une deuxième couche représentant des sables limoneux, des sables argileux, des argiles sableuses et des argiles compactes dont l'épaisseur ne dépasse pas $5 \mathrm{~m}$. Les résistivités observées varient de 400 à 69000 ohm.m ;

- une troisième couche montrant des résistivités faibles allant de 20 à $1400 \mathrm{ohm}$.m et d'épaisseur comprise entre 1 et $55 \mathrm{~m}$. Elle est constituée par l'alternance entre les sables moyens, les sables argileux et les sables grossiers. C'est la partie aquifère ;

- une quatrième couche représentant l'alternance entre les argiles compactes et les argiles sableuses dont les résistivités sont comprises entre 8000 et $84000 \mathrm{ohm}$.m. L'épaisseur de cette couche varie de 46 à $87 \mathrm{~m}$;

- la dernière couche, d'épaisseur infinie, montrant des résistivités faibles (350 à 550 ohm.m) ; il s'agit de l'alternance entre les sables moyens, les sables argileux et les sables grossiers. Cette couche constitue également le niveau aquifère. 

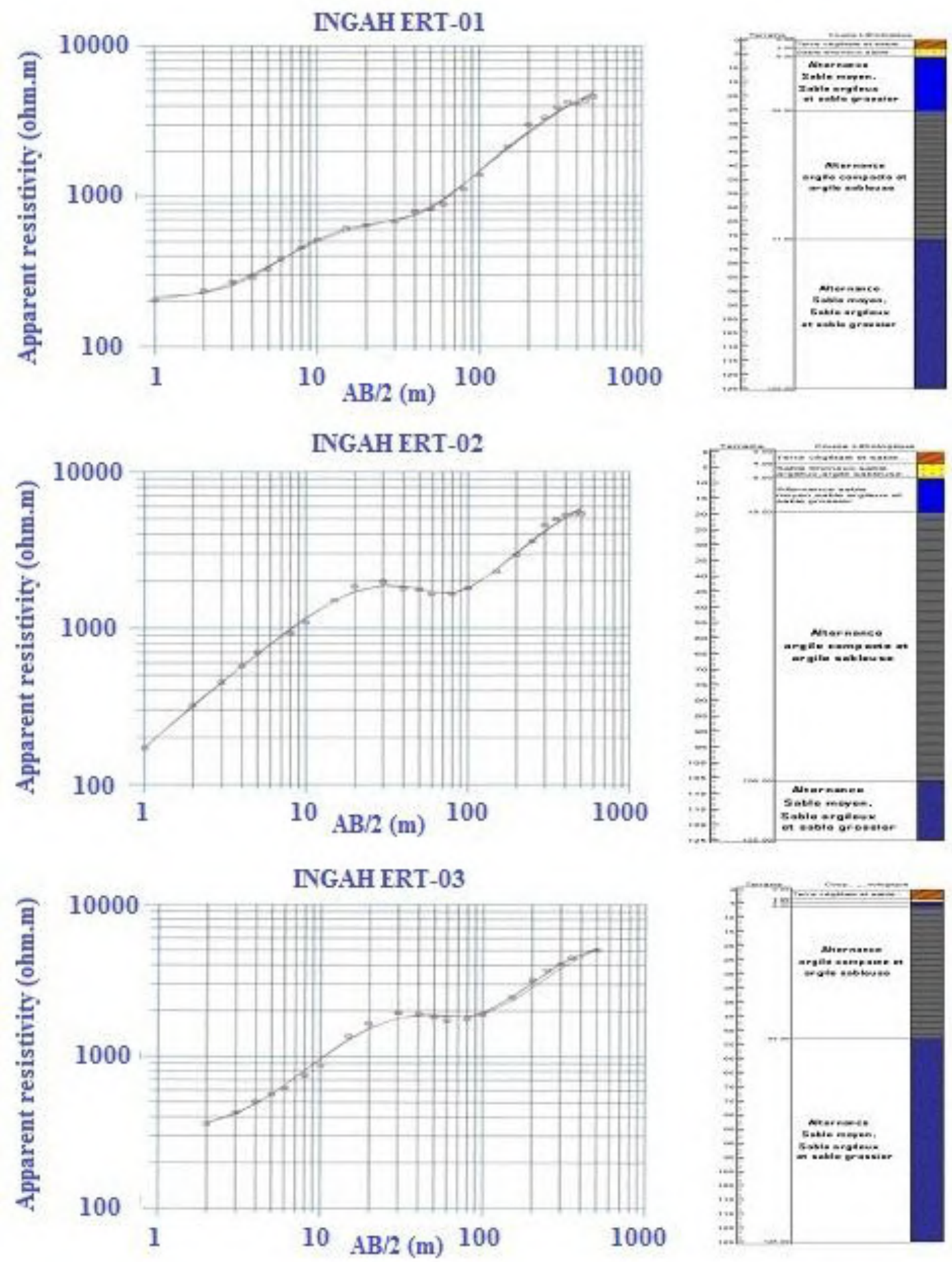

Figure 12. Variation de la résistivité en fonction de $A B / 2$ au site du village Ingah et modèle de sols associés (Fouroux, 2015). 

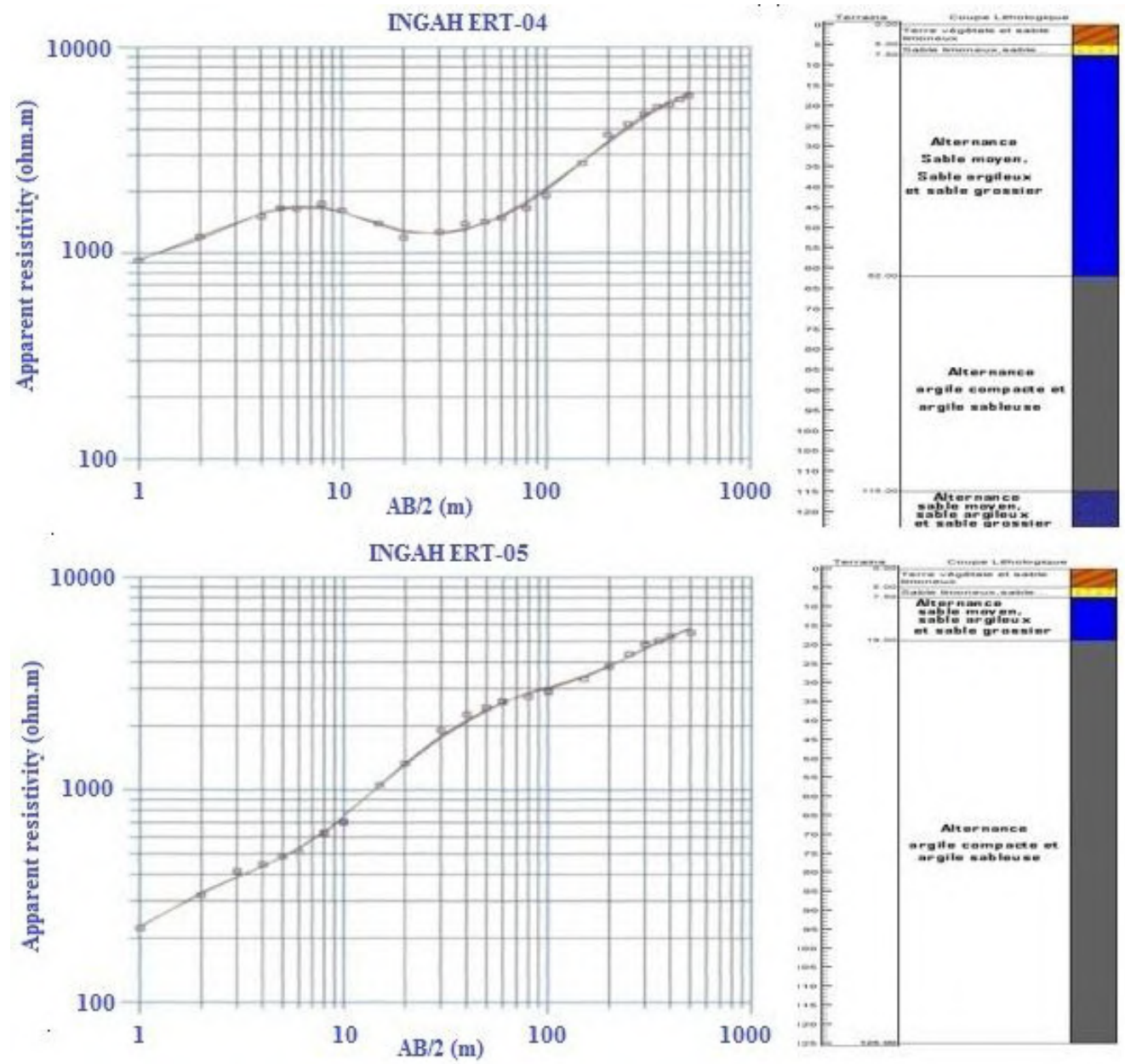

Figure 13. Variation de la résistivité en fonction de $A B / 2$ au site du village Ingah et modèle de sols associés (Fouroux, 2015).

\subsubsection{Profil géoélectrique du site du village Imvouba}

La Figure 14 illustre le profil géoélectrique du site du village d'Imvouba. Ce profil montre l'existence d'un niveau aquifère formé par l'alternance entre les sables moyens, les sables argileux et les sables grossiers. Le réservoir sablo-argileux est d'environ $300 \mathrm{~m}$ à l'aplomb du sondage de calage, dans un creusement du substratum et diminue progressivement en allant vers le SW où il atteint 6 mètres au niveau du sondage 5 . Il repose sur un substratum argileux d'épaisseur variant de 120 à $353 \mathrm{~m}$. La couverture sablo-limono-argileuse qui la surmonte, de manière continue a une épaisseur variant de 5 à $20 \mathrm{~m}$. Cette formation aquifère qui se biseaute vers le $\mathrm{SW}$ pourrait correspondre à un ancien lit d'un cours d'eau. Ce niveau qualifié de bon aquifère correspond à un aquifère superficiel alimenté par l'infiltration directe des eaux de pluie en raison de sa couverture sablo-limono-argileuse. 
Ce qui est en accord avec les travaux de Moukolo et al. (1992) et Seguin (2005).

La formation des grès, d'épaisseur infinie sur laquelle repose le substratum de l'aquifère correspond au socle. Il pourrait s'agir des grès de l'Inkisi sur lesquelles reposent les formations des plateaux Batéké conformément aux travaux de (Cosson, 1955).

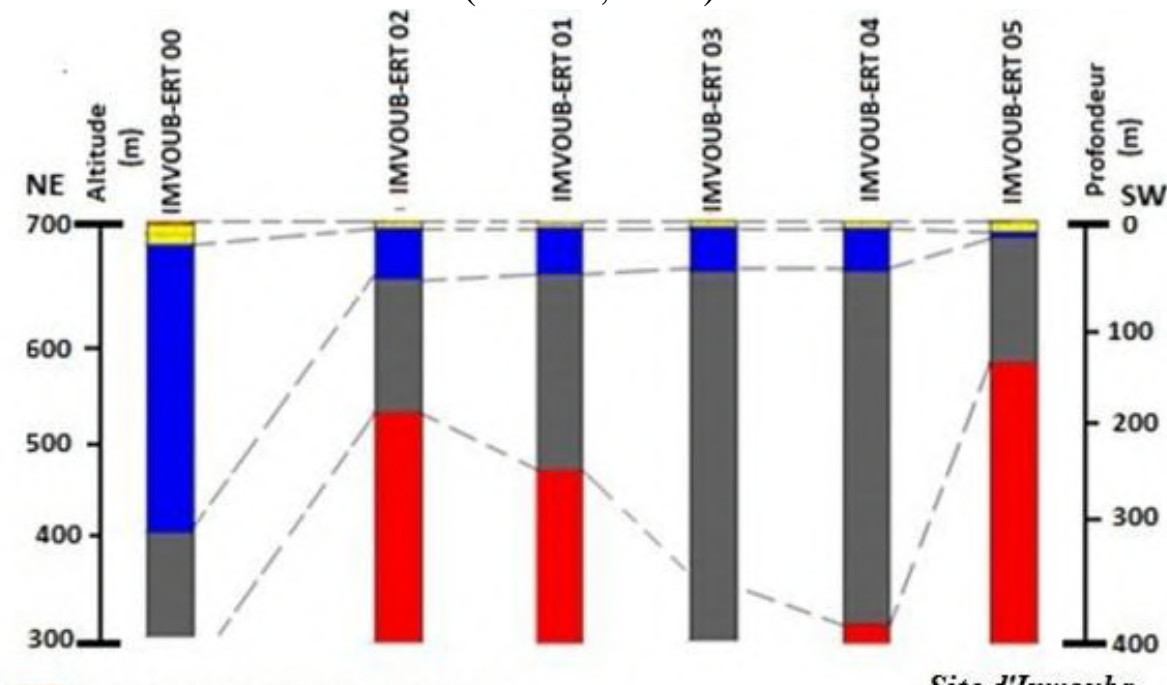

Terre végétale et sable limoneux

Site d'Imvouba

Sable limoneux, sable argileux, argile compacte et argile sableuse

Alternance sable moyen, sable argileux et sable grossier

Alternance argile compacte, argile sableuse

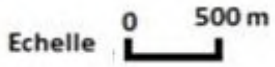

Grès

Figure 14. Profil géoélectrique du site du village d'Imvouba (Fouroux, 2015).

\subsubsection{Profil géoélectrique du site du village Ingah}

A Ingah, le profil géoélectrique (Figure 15) montre l'existence d'un système aquifère multicouche formé d'une nappe superficielle et d'une nappe profonde. Ces deux nappes sont formées par l'alternance des sables moyens, les sables argileux et les sables grossiers.

L'aquifère superficiel atteint $20 \mathrm{~m}$ d'épaisseur au NNE, dans un creusement du substratum au niveau du sondage 1, puis diminue progressivement en direction du sondage 3 où elle est d'environ $1 \mathrm{~m}$. Elle reprend de l'épaisseur à l'aplomb du sondage 4 dans un creusement du substratum où elle atteint son maximum (environ $55 \mathrm{~m}$ d'épaisseur) puis s'amaincit progressivement en direction du sondage 5 vers le SSE (environ 10 $m$ d'épaisseur). Ce réservoir repose sur un substratum argileux d'environ 90 $\mathrm{m}$ d'épaisseur. La couverture sablo-limono-argileuse coiffe le réservoir d'une manière continue. Son épaisseur est comprise entre 3 et $10 \mathrm{~m}$. Cette formation 
sablo-argileuse est un bon aquifère et est continuellement alimentée par l'infiltration directe des eaux de pluie.

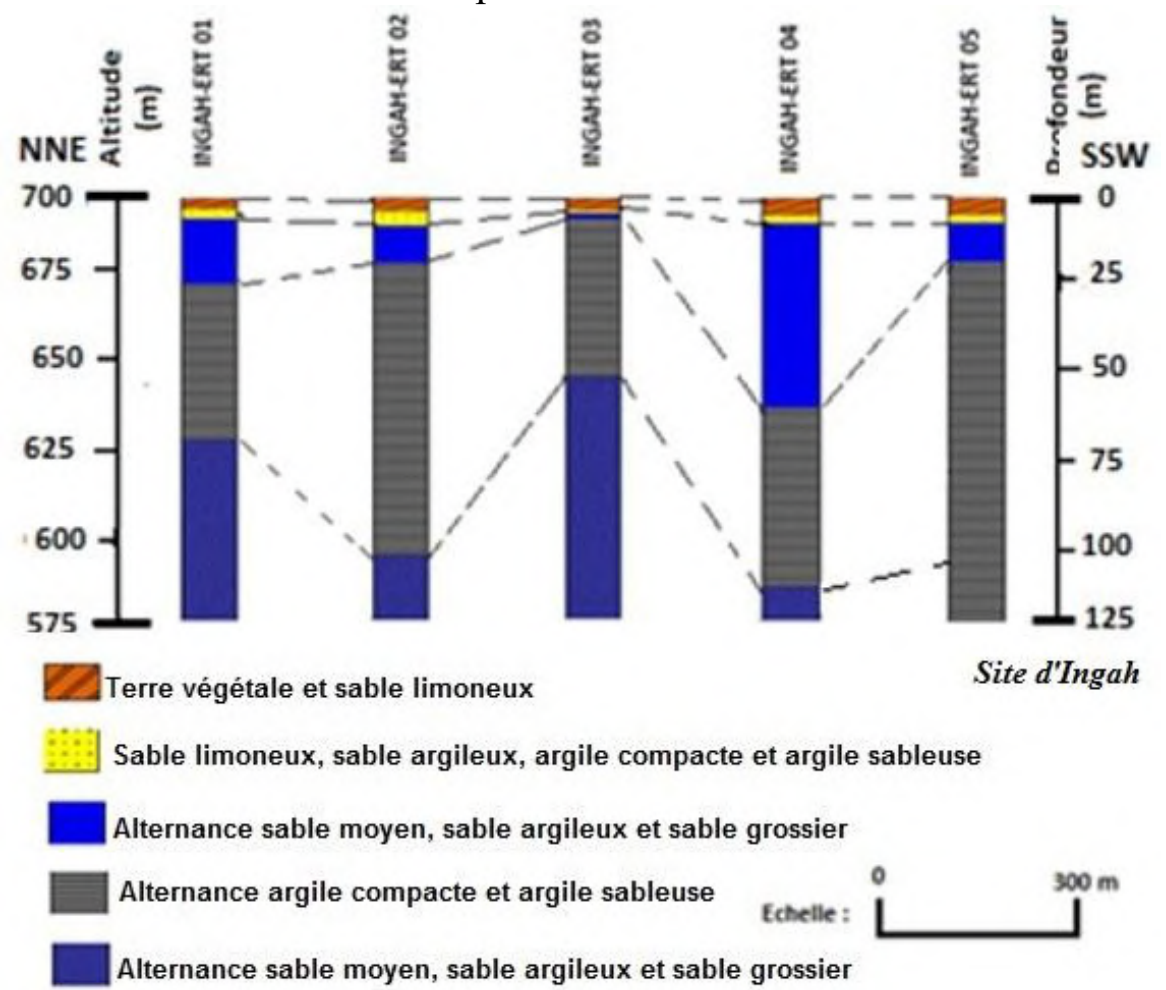

Figure 15. Profil géoélectrique du site du village d’Ingah (Fouroux, 2015).

L'aquifère profond d'épaisseur indéfinie est surmontée de manière continue par une formation alternant les argiles compactes et les argiles sableuses qui représentent le substratum du niveau aquifère superficiel. Leur épaisseur est comprise entre 46 et $87 \mathrm{~m}$. Malgré ses épaisseurs indéfinies, cette couche surmontée par des formations alternant les argiles compactes et les argiles sableuses est un bon aquifère. C'est un aquifère semi libre, alimentée à l'instar de l'aquifère superficiel par l'infiltration directe des eaux de pluie à cause de sa couverture plus ou moins perméable. Cette formation aquifère garantit plus ou moins la pérennité des ouvrages de captage en raison de sa couverture semi perméable, sablo-limono-argileuse. La formation alternant les argiles compactes et sableuses représentet d'une part le substratum du niveau aquifère superficiel et d'autre part le toît de l'aquifère profond. Cette couche permettrait dans certaines conditions des échanges d'eau entre les deux aquifères.

Les formations aquifères des villages Imvouba et Ingah, constituées par l'alternance entre les sables moyens, les sables argileux et les sables grossiers correspondent respectivement à des aquifères à nappe libre et 
multicouche. Ces couches aquifères sont alimentées par l'infiltration directe des eaux de pluie à cause de leur couverture sablo-limono-argileuse plus ou moins perméable et par des échanges hydriques avec les cours d'eau de la région. Elles garantissent un fonctionnement pluriannuel des ouvrages de captage en raison des conditions climatiques favorables (pluviosité abondante). Ceci confirme les travaux de Moukolo et al. (1992) et Seguin (2005) dans les plateaux Batéké en général et dans le plateau de Mbé en particulier.

Après l'analyse des résultats issus de l'interprétation des courbes des sondages électriques qui présentent une certaine similitude d'un site à l'autre et considérant les modèles géoélectriques des différents sondages électriques d'une part et les résultats des forages déjà réalisés dans la zone d'étude d'autre part, l'implantation des forages se fera à des profondeurs prévisionnelles de 250 à $300 \mathrm{~m}$ pour les sites des villages Imvouba et Ingah.

\subsection{Forages de reconnaissance et d'exploitation}

\subsubsection{Coupes lithologiques et techniques des forages d'eau}

A Imvouba comme à Ingah, la foration a débuté par la réalisation d'avant-trous en diamètre de 17''1/2 jusqu'aux profondeurs respectives de $100 \mathrm{~m}$ et $69 \mathrm{~m}$. Ces avant-trous ont été équipés en tube de soutènement API 13 '' $3 / 8$ pour isoler les terrains tendres. Après isolation des terrains tendres, la foration a été poursuivie en reconnaissance au rotary à la boue en diamètre 9', $5 / 8$ jusqu'à $317 \mathrm{~m}$ à Imvouba et $295 \mathrm{~m}$ à Ingah (Figure 13). Enfin un alésage au 12''1/4 a été effectué pour la pose de l'équipement (Fila, 2016).

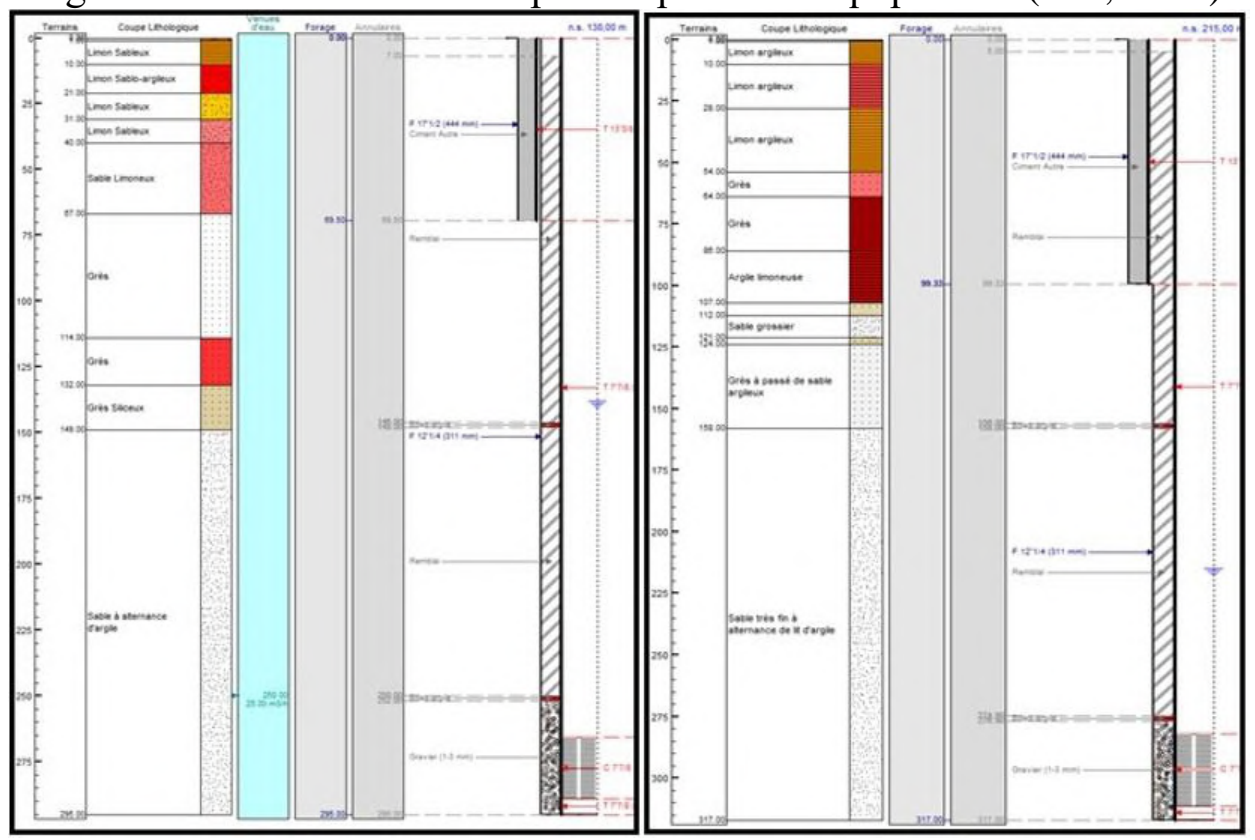

Figure 13. Coupes lithologiques et techniques des forages d'Ingah et d'Imvouba (Fila, 2016). 


\subsubsection{Identification des formations aquifères}

L'aquifère superficiel atteint $20 \mathrm{~m}$ d'épaisseur au NNE, dans un creusement du substratum au niveau du sondage 1 puis diminue progressivement en direction du sondage 3 où elle est d'environ $1 \mathrm{~m}$. Elle reprend de l'épaisseur à l'aplomb du sondage 4 dans un creusement du substratum où elle atteint son maximum (environ $55 \mathrm{~m}$ d'épaisseur) puis s'amincit progressivement en direction du sondage 5 vers le SSE (environ 10 $\mathrm{m}$ d'épaisseur). Ce réservoir repose sur un substratum argileux d'environ 90 $\mathrm{m}$ d'épaisseur. La couverture sablo-limono-argileuse coiffe le réservoir d'une manière continue. Son épaisseur est comprise entre 3 et $10 \mathrm{~m}$. Cette formation sablo-argileuse est un bon aquifère et est continuellement alimentée par l'infiltration directe des eaux de pluie (Fila, 2016).

La formation des grès, d'épaisseur infinie sur laquelle repose le substratum des aquifères profonds correspond au socle. Il pourrait s'agir des grès de l'Inkisi sur lesquelles reposent les formations des plateaux Batéké conformément aux travaux de (Moukolo et al., 1992).

\section{Conclusion}

La prospection hydrogéologique et géophysique entreprise dans le plateau de Mbé a permis de déterminer la qualité des eaux souterraines et de surface exploitées dans le plateau de Mbé et les niveaux aquifères favorables à l'implantation des forages productifs. Elle montre la dominance du faciès chimique bicarbonaté et calcique. Ces eaux sont très faiblement minéralisées avec des valeurs de conductivité électrique comprises entre 0,12 à 105,7 $\mu \mathrm{S} / \mathrm{cm}$. Les valeurs de $\mathrm{pH}$ sont comprises entre 4,85 et 7,23 et les eaux sont dans l'ensemble acides à neutres. Dans ces conditions, l'alcalinité des eaux serait essentiellement contrôlée par les bicarbonates. La bonne qualité physico-chimique des eaux souterraines et de surface est remise en cause par la présence de certains éléments comme le fer total, le manganèse, les phosphates et l'aluminium à des teneurs élevées dépassant parfois les concentrations maximales admissibles pour l'eau de boisson définies par l'OMS.

Les résultats des sondages électriques montrent au village Imvouba un creusement du substratum correspondant probablement à un ancien lit du cours d'eau. Par contre à Ingah, les résultats des sondages électriques montrent l'existence d'un système aquifère multicouche formé d'une nappe superficielle et d'une nappe profonde.

En conséquence, les formations géologiques en présence dans les plateaux Batéké en l'occurrence dans le plateau de Mbé renferment un système aquifère multicouche à nappe libre bien alimenté par l'infiltration directe des eaux de pluies (1600 à $2500 \mathrm{~mm} / \mathrm{an}$ ) et par des échanges hydriques avec les cours d'eau de la région. Ainsi, l'aquifère profond est continuellement en 
charge et garantit un fonctionnement pluriannuel des forages d'eau. Cependant la maîtrise technique et la méthodologie de foration et d'équipement jouent un rôle capital dans la productivité des ouvrages de captage. Les travaux de réalisation des forages devraient être suivis par un hydrogéologue qui jugera sur place de l'arrêt ou de la poursuite de la foration en fonction des réalités rencontrées sur le terrain (avancement de l'outil de forage, lithologie et venues d'eau observées) et des débits prévisionnels.

Enfin, de nombreuses études réalisées depuis plusieurs années dans la recherche des eaux souterraines, ont montré que la géophysique est devenue un outil quasi indispensable mis à la disposition de l'hydrogéologue. Son application permet d'établir un rapport de synthèse beaucoup plus complet, compte tenu des données aussi bien superficielles que profondes et de localiser au mieux les premiers ouvrages de reconnaissance avec une probabilité maximale de réussite.

\section{References:}

1. Abdel Galil A.H., Essam A. El-M., Sultan A. S., Talaat M. R., Ahmed A. El k., \& Shokry A. S. (2015). Groundwater exploration using resistivity and magnetic data at the northwestern part of the Gulf of Suez, Egypt. Egyptian Journal of Petroleum, vol. 24(3), pp. 255-263.

2. Assi-Kaudjhis C., Digbehi Z. B., Roche E. \& Lezine A.M. (2010). Synthèse sur l'évolution des paléoenvironnements de l'Afrique occidentale atlantique depuis la fin de la dernière période glaciaire. Influences climatiques et anthropiques. Géo-Eco-Trop, 34 : pp. 1-28.

3. Babet V. (1947). Exploration de la partie méridionale des plateaux Batéké. Bull. du Service des Mines de 1'A.E.F., n 3, pp. 31-56.

4. Banton O., Bangoy L.M., Chevalier S., Houenou P., Lafrance P. \& Rivard C. (1997). Hydrogéologie : Multiscience environnementale des eaux souterraines. Presses de l'Université du Québec/AUPELF, 460 p.

5. Bauer H., Callec Y., Prognon F., Issautier B., Schroetter J.M., Thiéblemont D., Nganga Lumuamu F. \& Dah Tolingbonon R.H. (2015). Notice explicative de la carte géologique de la République du Congo au 1/200000, Feuille Mbé-Ngabé. Éditions BRGM, 113 p.

6. Cosson J. (1955). Notice explicative sur les feuilles Pointe-Noire et Brazzaville. Carte géologique de reconnaissance au 1/500.000. Dir. Mine. Géol. A.E.F. Brazzaville, 56 p.

7. Dadet P. (1969). Notice explicative de la carte géologique de la République du Congo Brazzaville au 1/500 000. Zone comprise entre les parallèles $2^{\circ}$ et $5^{\circ}$ Sud. Mémoire BRGM, France, 70, 103 p.

8. Destieux F. (1993). Notice explicative de la carte géologique du Congo au 1/10000000, MINE, ISBN 062-1599, 1-3, 27 p. 
9. Fila D.S. (2016). Suivi de l'exécution de deux forages d'eau pour la desserte d'eau par mini réseaux dans les villages d'Ingah et d'Imvouba dans le district de Ngabé (Département du Pool). Mémoire de Master de Géosciences, Faculté des Sciences et Techniques, Université Marien NGOUABI de Brazzaville, 59 p.

10. Fouroux M. A. (2015). Prospection hydrogéologique et géophysique pour l'implantation des forages d'eau dans le plateau de Mbé (Département du Pool, Congo). Mémoire de Master de Géosciences, Faculté des Sciences et Techniques, Université Marien NGOUABI de Brazzaville, $91 \mathrm{p}$.

11. Giresse P. (1990). Paleoclimatic and structural environment at the end of the Cretaceous along the western flank of the Congo Basin, with application of underground microdiamonds around Brazzaville. Journal of African Earth Sciences 10: pp. 399-408.

12. OMS/WHO (2017). Directives de qualité pour l'eau de boisson, $4{ }^{\text {ème }}$ édition Intégrant le premier additif. ISBN 978-92-4-254995-9. 564 p. https://creativecommons.org/licenses/by-nc-sa/3.0/igo.

13. Miyouna T., Malounguila D.M., Essouli O. F., Ndembé-Ndembé A. J., Moussiéssié J., Kinga Mouzéo, \& Boudzoumou F. (2016). Etude paléoenvironnementale des dépôts détritiques de la formation de couverture du bassin côtier du Congo. Rev. CAMES-Vol. 04 NUM.01. *ISSN 2424-7235.

14. Mohamaden I. I. M. \& Ehab D. (2017). Application of electrical resistivity for groundwater exploration in Wadi Rahaba, Shalateen, Egypt. NRIAG Journal of Astronomy and Geophysics, Vol 6(1), pp. 201-209.

15. Moukolo N., Samba-Kimbata M.J., Mpounza M., Nzalabaka J., Maziézoula B. \& Ossété J.M. (1992). Hydrogéologie du Congo, document du BRGM n ${ }^{\circ}$ 210. Editions du BRGM (France), 142 p.

16. Moukolo N. (1992). Etat des connaissances actuelles sur 1'hydrogéologie du Congo. Journal hydrogéologie, 1-2 : pp. 47-58.

17. Sabata B.C. (2017). Hydrochimie des eaux souterraines et de surface du plateau de Mbé (Département du Pool). Mémoire Master de Géosciences, Université Marien NGOUABI de Brazzaville, Faculté des Sciences et Techniques, $50 \mathrm{p}$.

18. Serrane M., Bruguier O., \& Moussavou M. (2008). U-Pb single zircon grain dating of Present fluvial and Cenozoic Aeolian sediments from Gabon: consequences on sediment provenance, reworking, and erosion processes on the equatorial West African margin. Bulletin Société Géologique de France, t 179, N¹, 29-40.

19. Seguin J. J. (2005). Projet Réseau SIG-Afrique. Carte hydrogéologique de l'Afrique à l'échelle du 1/10 M, BRGM/RP - 54404 - FR. 
20. Selim A. (2009). Geophysical Tools Using in Groundwater Exploration. Doi : 10.13140/RG.2.2.20756.30085.

21. Steven, M.B., Howard, S.B., Zaruhi, R.M., Liane, C.R.T., Michael, W. T., Richard, A.W., Lewis, S.H., \& Yund, C. (2008). Drinking Water Security and Public Health Disease Outbreak Surveillance. Johns Hopkins APL Technical Digest, Volume 27, Number 4, pp. 403 - 410.

22. Thieblemont, D. (2013). Evidence for an aeolian origin of the Holocene lateritic surface cover of Gabon. Quat. Int. 296, 176-197.

23. Robert Anechana, Reginald Mensah Noye, Aboagye Menyeh, Evans Manu, \& Collins Okrah (2015). Electromagnetic Method and Vertical Electrical Sounding for Groundwater Potential Assessment of Kintampo North Municipality of Ghana. Journal of Environment and Earth Science, Vol.5, No.12, , pp. 9 - 16.

24. Rodier J. (1996). L'analyse de l'eau, eaux naturelles, eaux résiduaires, eaux de mer. Edition Dunod, 1434 p. 